\title{
City Data Plan: The Conceptualisation of a Policy Instrument for Data Governance in Smart Cities
}

\author{
Lucia Lupi ${ }^{1,2}$ \\ 1 DIST-Interuniversity Department of Urban and Regional Studies and Planning, \\ Polytechnic and University of Turin (IT), 10129 Torino, Italy; lucia.lupi@polito.it \\ $2 \mathrm{KMi}-$ Knowledge Media Institute, The Open University (UK), Milton Keynes MK7 6AA, UK
}

Received: 29 June 2019; Accepted: 11 August 2019; Published: 13 August 2019

\begin{abstract}
This paper presents the conceptualisation of the City Data Plan, a data governance policy instrument intended to connect the production and use of urban data in a comprehensive and evolutive long-term strategy aligned with city development goals. The concept of the City Data Plan had been elaborated by taking into account current issues related to privacy and manipulation of data in smart city. The methodological approach adopted to define the nature of a City Data Plan is grounded on the conceptual and empirical parallelism with corporate data governance plans and general urban plans, respectively aimed to regulate decision-making powers and actions on data in enterprise contexts, and the interests of local stakeholders in the access and use of urban resources. The result of this analytic process is the formulation of the outline of a City Data Plan as a data governance policy instrument to support the iterative negotiation between the instances of data producers and data users for instantiating shared smart city visions. The conceptualisation of the City Data Plan includes a description of the multi-stakeholder organisational structures for the city data governance, cooperation protocols and decision areas, responsibilities assignments, components of the plan and its implementation mechanisms.
\end{abstract}

Keywords: city data; data plan; urban plan; data governance; smart city; city development

\section{Introduction}

The production of data in urban environments is constantly increasing in the last few years due to the deployment of smart city technologies and a generalised awareness of the value of data as "engines for the new economy" [1-3]. Nevertheless, the unregulated collection and exploitation of data produced in cities (city data) is fostering the rise of significant political and social concerns. Indeed, data are not only resources but also a source of conflicts in the relationships between government and citizens, technology providers and users-clients, organisations capitalising the access to information, and people excluded from the benefits generated from their data.

National governments and supranational authorities are increasingly worried about the manipulation of information on social media and the destabilising consequences of this phenomenon at a local and global scale. Local governments are starting to question their dependence from the services provided by the major ICT (Information and Communication Technologies) global corporations, that currently are the primary beneficiaries of the value generated by city data. Activist groups and intellectuals already brought to light fears and risks linked with the progressive setting up of a surveillance society, contributing to sensitise civil society on the issues of privacy and equity in the access and use of data [4-6].

The most significant efforts done so far to face these political and social problems had been mainly focused on contrasting the indiscriminate commercialisation of data. The most significant example is the EU General Data Protection Regulation. The GDPR [7] represents a substantial change in the 
privacy protection of European citizens accessing or traced by digital systems. At the local level, institutional initiatives such as "Data as Commons" promoted by the municipality of Barcelona [8] are a sign in the direction of empowering citizens to consider data as a shared resource to improve city services. Another example is the "TADA Manifesto", elaborated by activists in Amsterdam [9], and focused on defining the ethical uses of data that respect the principles of transparency, privacy control, inclusiveness and openness to local communities.

These types of initiatives are oriented to contrast the negative consequences of the availability of data on individuals, limiting the risks of privacy violations and public exposure of sensitive information. However, these initiatives do not address the structural problems and the issues linked to the production of city data. In this sense, two of the open challenges for the governance of city data concern:

(a) managing the uses of data generating social conflicts in the city, such as inequalities and unbalanced power relationships between who exploit data and who are exploited for data and through data

(b) moving the focus of data policies from individuals to organisations representing the diversified range of stakeholders operating in the city, such as public and private institutions, small and medium businesses, and local communities.

The underlining questions to address these two challenges are:

- How to facilitate a sustainable redistribution of the benefits associated with the availability of an unprecedented among local stakeholders?

- How to frame the production and use of city data into a comprehensive vision of social and economic development for the city?

This work explores these questions adopting a design-oriented approach informed by urban planning practices applied to similar challenges in the city context. Indeed, the core of urban planning practices is the design and implementation of policy and regulatory instruments concerning the redistribution of the surplus connected to the creation and transformation of urban resources [10]. Even though city data had been not yet formalised as a matter of urban planning practices, their status of essential resources in urban activities made possible to reason on them precisely like every other asset in urban environments. Another distinctive aspect of urban planning theory and practice is dealing with the complexity of contextual, technological, normative and social constraints to compose competing goals and heterogenous instances into a coherent vision [11,12]. Therefore, urban planning practices can be explored to compose the various perspectives of different local stakeholders into a coherent governance model for the production and use of city data.

It is important to highlight that urban planning is usually considered as a passive "recipient" of technologies and city data. The critical assumption of this work is instead that urban planning practices can actively inform future solutions to address data-related social conflicts in the city context. Indeed, smart city literature indicates urban planning as one of the most promising sectors for connecting the "data layer" of cities to the development of digital services for capitalising tangible and intangible resources in the city $[13,14]$. However, even recent smart city frameworks centred on social and governance aspects in the relationship between cities and technologies [15-17] tend to ignore that the fundamental scope of urban planning is orienting the economic growth and socio-cultural development of the city, in addition to its physical transformation [18]. Secondly, smart city strategies so far underexplored the strategic approach commonly adopted in urban planning to orient the city growth and development by using spatial arrangements as proxies for social and economic interventions on local resources [19]. Lastly, the implementation of smart city initiatives is still grounded on the disconnection between the deployment of new technologies in the city and the nature of local needs [20]. On the contrary, urban planning in the last century supported the modernisation of society and cities precisely by grounding its practices in the knowledge of the context and local needs as the driver for generating social and economic development [21]. 
Building on these considerations and against the mainstream passive framing of urban planning in smart cities, this work points out instead the potentialities of the inputs coming from urban planning practices to actively design new policy instruments for a multi-stakeholder governance of city data.

This paper presents the City Data Plan as a design concept aimed to envision the management of the production and use of city data by local stakeholders in compliance with negotiated goals and vision of the city. These goals are expressed in the form of a plan including rules and actions concerning the use of available data, the production of data for enabling future actions, and the cooperative mechanisms for the generation of public value from city data.

The concept of the City Data Plan had been drafted by integrating the analysis of the logics and measures characterising urban plans (focused on the management of urban resources, but not on data as city assets) with the analysis of the structures and protocols characterising data governance plans in enterprise context (focused on data, but not on social or political aspects relevant in the city context). Therefore, the City Data Plan results from the hybridisation of these two policy instruments, urban plans and corporate data governance plans.

The remaining of the paper is structured as follows.

- Section 2 provides a short overview of the key topics and issues concerning the governance of data in smart city literature.

- Section 3 explains the methodological approach adopted for defining the City Data Plan through the theoretical analysis of the concepts describing corporate data governance plans and urban plans.

- Section 4 reports on the analytic process to conceptualise the City Data Plan.

- Section 5 presents the concept of the City Data Plan, summarising its fundamental characteristics in relation to the constraints of the problem of the governance of city data, as emerging from related works reviewed in Section 2.

- Section 6 describes three examples of urban applications of the City Data Plan in the specific context of the city of Milton Keynes (UK).

- Section 7 discusses potentialities, limitations and open challenges of this type of policy instruments respect to the constraints of the problem of the data governance in the city.

- Section 8 concludes the paper by highlighting the contribution of the work to the domain of urban planning research and practice.

\section{Related Works}

The governance of data produced and used in cities is a topic still underexplored. Earlier studies focused on investigating nature, limits and applications of data-driven actions in urban governance $[2,14,22-26]$, or rather how the access to data can inform better governance decisions and policies. Critical perspectives on smart cities constitute an essential complement to these earlier studies for exploring in-depth the hidden concerns related to data availability and governance of data, going beyond the aspects related to a data-driven urban governance.

This section examines the nature of data in smart cities from a critical perspective on data governance. In particular, the literature review focuses on the issues associated with specific uses or misuses of data in the relationships between different actors in the city (see Figure 1), and then discusses the strategies and potential solutions proposed in the literature to address these issues.

Until two decades ago the production of data concerning city activities was focused on "small data", generated by public administrations and agencies as part of their activities (e.g., census data). Smart City technologies triggered a paradigmatic shift in the capability of dynamically tracking changes in the physical environment of cities and people's behaviours through the collection and analysis of "big data". Small Data conventionally includes complex elaborations of information organised in textual and visual forms, such as in reports or annotated tables, legible and understandable by humans. Differently, big data are data automatically generated from sensors and devices monitoring the urban 
fabric and its infrastructures, through geo-localisation systems recording people's movements, and by aggregating metadata and contents from social media platforms [27].

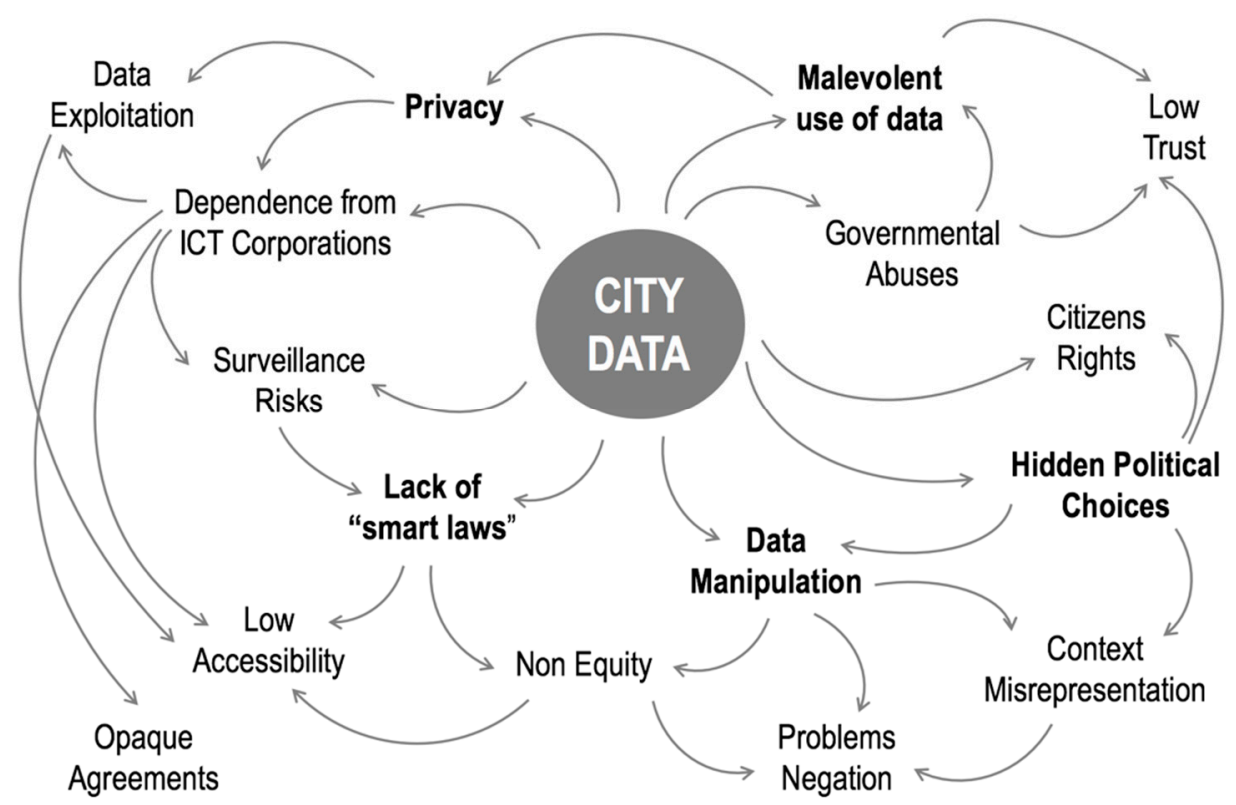

Figure 1. Map of the key issues associated to city data and their governance in the smart city literature (source: author's elaboration).

The first type of these data, those from sensors, are defined by Kitchin as "urban operational data" [28]. They are usually assumed as objective traces of facts (e.g., the status or performances of infrastructures) and considered just as functional products aimed to support public or private agencies in the efficient real-time management of planning operations, local services, and infrastructures [29]. However, the supposed rational data-driven decision trees implemented in these management processes are never isolated from the political, social and organisational constraints bounding the decisions and actions of public or private agencies $[23,26,30]$. For that reason, even urban operational data should be considered as part of a "data assemblage" [27] in which datasets and technologies to generate and access data are strictly dependent on the context in which data are intended to be used. Therefore, data collected in cities are always the result of specific systems of thought, organisational or political settings, economic factors, and choices about what needs to be traced through data [27]. The social, political, economic components of the "data assemblage" impact on what types of data are collected, where, from whom, and for what purpose, independently from the assumed objectivity of data.

Sensors to monitor air quality in cities provides a meaningful example of this point. Data about the pollution levels or the density of a specific pollutant provide objective information, as recorded by sensors. Nevertheless, the collected data are determined by the choice of installing those sensors in a certain area instead than another, and this choice is linked to the goal that the collected data are intended to serve. For instance, placing pollution detectors close to the industry district can help in proving that the air quality in a city is very low and facilitate the adoption of extraordinary measures to fight apparently critical levels of pollution. On the opposite side, the same sensors can be placed close to green areas to show that recently approved policies to improve air quality in the city are already effective. These two extreme cases of instrumental and manipulative use of data from pollution detectors illustrate the tension between the objectivity of data and their partiality, as well as the difficult legibility of the hidden factors determining the content of data in themselves.

Differently from urban operational data, data from social media platforms provide an account of points of views, perspectives, positions that traditional public surveys do not capture. The massive adoption of social media platforms (by almost every age group and socio-economic population 
segment) provides unprecedented coverage to understand public opinion formation, personal and collective preferences, social phenomena in real-time. However, it is difficult or risky to consider these sources as reliable representations of local societies and their needs. Indeed, as demonstrated by recent studies, social media platforms have engagement mechanisms that tend to support only certain users' behaviours [31,32], and types of communications reflecting only very partially individual and collective positions [33]. As a consequence, it is problematic to assess the correspondence between phenomena traced in social media and real needs anchored to local situations and specific city contexts.

Despite this fact, data from social media platforms are usually exploited by public and private actors to develop "geodemographic profiles" [34] for a variety of operations that can benefit from targeting customers and end users (e.g., advertisement or sensibilisation campaigns). These operations can also include illegitimate actions oriented to manipulate the public opinion by leveraging on social weaknesses and fears emerging from data.

The two major issues associated with the governance of city data, both urban operational data and social media data, concern:

- The manipulation of data

- The indiscriminate use of data to control social phenomena or groups

Bowker [35] argues that city data can help to understand social problems and economic fragilities and actively operate to address them. At the same time, he points out that data can also be used to negate the existence of these problems, with the effect of consolidating urban inequalities that persist in the real city. This position is supported by other recent works $[22,29,36]$ highlighting the manipulation of data as the effect of having the resources to influence the data collection and analysis process and the opportunity to benefit from the data manipulation directly.

Lyon [37] critically analyses the social and political consequences of the availability of data in terms of their capability to predict future events, based on inferences from data on the past and present phenomena. As mentioned before, these data are always partial representations of a context and their relevance to outline the future status of affairs should be carefully evaluated.

For instance, Leszczynski [36] highlights that city data can be actively used for preventing social disorder and controlling the generation of a specific type of information accessible to the public. To this regard, Leszczynski warns from the risks of timely and disproportioned reactions to data flows generated on social media relying on the inputs coming from the analysis of past events. These reactions can be oriented indeed to suppress social conflicts or political oppositions considered dangerous from the perspective of law enforcement authorities, but not necessarily by civil society and in a democratic context.

Considering more ordinary situations, Finch and Tene [38] underline that the availability of fine-grained data can easily support the use of data with discriminatory intents by insurance companies, banks and mortgage agencies, often with severe social consequences if systematically replicated at large scale.

The manipulation and malevolent use of data in the city context are sensitive governance issues reflecting the unbalanced power between organisations controlling city data and other social groups and organisations excluded from decisions and operations on city data. Usually, the organisations controlling the production and use of city data are technology providers and public authorities. On the opposite side, the other actors in the city data ecosystem are:

- The subjects from which data are collected (usually citizens and city services customers)

- The intended users of data (other organisations, public, private or non-profit)

- Knowledge professionals and media that communicate through data

This unbalanced power, given by the priority or exclusive access to city data, is the effect of a normative lack of regulations and policies establishing in operational terms the acceptable uses of data to pursue objectives not in conflict with the public interest. Metaphorically, this situation is similar to a 
coalition of groups controlling goods and services supply driven by their own goals instead of being oriented by the citizens' needs.

The lack of "smart laws" for smart technologies regulating also the data produced by these technologies is a well-known problem. It started to be explored only very recently for elaborating appropriate solutions to prevent or mitigate potential misuses of data [39].

Finch and Tene [38], working in this new research strand, indicate four potential strategies and approaches to improve the governance of data produced and used in cities by smart city technologies:

(a) Getting smart city technologies more familiar to the public through dissemination and public information activities

(b) Enforcing the anonymisation of data and contrasting data crossing procedures for re-identification

(c) Enhancing the transparency in the use of data for city operations by governmental authorities

(d) Moving from data as objects to data as a "plus" of digital services through the "data featurization".

This fourth strategy corresponds to the practice of "making data a consumer-side feature of products and services" [38]. In other words, the proposal is collecting and delivering information to the users for their direct benefit and purposes. The main limitation of this strategy is its focus on the benefits of individual users, that makes unpracticable reaching higher goals of redistributing the benefits of data collected by smart city technologies to community groups or local businesses and other organisations operating in the city. Even in the case in which data featurization would become a common practice for commercial applications, the governance of these data remains uncertain and determined only by market-driven relationships between individual users and technology company acting as data and data-driven service providers. In this schema, the governance of data at the city level still remains unconnected to city development strategies, without enabling synergies within the city data ecosystem.

Differently from Finch and Tene, Rabari and Storper [40] highlight the importance of making city data available to local organisations. They frame city governance as a multi-level dialogue between state and non-state actors (privates, non-profit organisations, local communities) that should be also extended to city data and digital technologies. Rabari and Storper suggest that establishing an open governance of city data would improve participatory and democratic processes, the coordination among public agencies, and better relationships between local governments and other social forces in the city. To this regard, Ting and Williamson [41] made explicit that one of the critical aspects to be taken into account for enabling a collaborative governance of data produced at the urban level is the relevance of data shared across organisations for their respective decisions making processes and activities. However, these authors do not indicate approaches or specific solutions to pursue these aims.

In the emerging landscape of potential solutions for the governance of city data in smart cities, one of the first attempts to outline a comprehensive strategy to connect urban governance and city data in procedural terms is documented in the report published by the Government Data Forum for Irish cities and linked to the project "The Programmable City" [24]. The study proposes four types of institutional structures aimed to primarily address privacy and security concerns regarding smart city technologies within a policy framework. These four institutions are:

(1) A Smart City Advisor Group including representatives of all the relevant stakeholders involved in the city data ecosystems or in local activities of public relevance (such as community organisations). The aim of the Advisory Group is aligning the smart city agenda and city development plans in terms of goals and resources.

(2) A Smart City Governance, Ethics and Security Oversight Committee in charge of supervising the implementation of the strategies of the advisor groups. The Committee establishes what data are to be generated, how they are analysed, processed and transferred to third parties, their licensing and potential applications or acceptable uses.

(3) The core privacy/security team implementing day-by-day the plan established by the oversight committee. The team is responsible for testing and assessing the systems deployed in the city, 
connecting city departments and companies to smart city initiatives, and communicating smart city policies to the public.

(4) The City Computer Emergency Response Teams activated in case of cybersecurity incidents or problems to smart city technologies.

This institutional schema is aimed to build a socio-political infrastructure to enable a multi-stakeholder negotiation and the consensus about the city development visions to be pursued through smart city initiatives. The dialogue and cooperation among different organisation are recognised as foundational for also overcoming the major concerns about privacy and malevolent use of data by establishing transparent rules under shared political and social assumptions made public and legible to all the social forces in the city. These rules specifically concern the choices for the production and use of data by taking into account the plurality of interests associated with city data. The two aspects that remained unaddressed in this schema concern:

- The type of normative and technological instruments to support the interdependent activities of the proposed institutional structures.

- The potential measures to deal with variable levels of commitment of politics and local actors to the continuous implementation of a common strategy for the production and use of city data.

The overview of the related works on the governance of city data highlighted that the fundamental issues are the potential manipulation and misuses of data and their consequences. The literature pointed out also the lack of widely accepted protocols to establish a governance of city data that can reduce these risks and maximise the positive outcomes related to the availability of data for society. In this landscape, city data governance arrangements are elaborated case by case between public authorities and technology providers, influenced by contingent situations, and do not necessarily cover the aim of linking the production and use of city data to the development strategies of cities. A concrete alternative to this practice is proposed by the measures contained in "The Programmable City" report [24] grounded on the acknowledgement of the complex nature of data, inseparable from their political and social context.

Building on this background, it is possible to synthesise that the type of solutions to address the examined issues for the governance of city data met the following meta-requirements. The "desirable solutions" should be:

(1) Oriented to balance the power of data producers and primary users (technology providers and public authorities) respect to other actors involved in the city data ecosystem as data collectors, secondary users, data subjects (i.e., citizens, local communities, businesses, non-profit organisations)

(2) Based on negotiation and consensus among city stakeholders on the contents of city data policies

(3) Managed through transparent and explicit rules for the production and use of data that distinguish between legitimate and illegitimate uses of data and reveal the rationale of data-centred operations

(4) Built to connect the production and use of city data to local needs for effectively exploiting data to address real problems

(5) Reactive and responsive to the evolution of the city data ecosystem through flexible mechanisms that can be refactored over time to achieve new goals, include new actors, integrate new resources.

\section{Approach to the Conceptualisation of the City Data Plan}

The five meta-requirements for city data governance solutions emerging from the literature had been assumed as the starting point of this work. The exploration of tools, instruments, policies meeting these requirements followed a design research approach that informed the research design.

The choice of adopting a design research approach implied an investigation process driven by a "solution-focused strategy" grounded on understanding the problem constraints from the literature and practice. A design research approach takes an opposite stand respect to a "problem-focused 
strategy", more common in natural and social sciences. Problem-focused investigations are oriented to decompose and analyse a problem with the purpose of describing the problem in itself, but not necessarily elaborating alternative solutions to a current state of affairs. Differently, solution-focused investigations are aimed to outline a possible (and feasible) alternative to the current state of affair and to address the problem, not describing it [42].

The first step of the design research process consisted in reframing the problem to be addressed in operational terms, or rather in a form that allows the definition of an intervention space and practical reasoning on social, operational and technical constraints.

Considering that the output of a design research process is a design (material or conceptual as in this case), the three focal points to reframe a problem are the intended Users of the proposed solution, the Context in which the solution will be placed, and the central Object of the solution. In this study, the three focal points are:

- The different classes of stakeholders operating in the city as Users of the solution. They include:

(a) Local governments (political and administrative authorities at the city level)

(b) Public agencies (administrative and operational unit for the provision of public services at local, regional and national level)

(c) Business sector (industry, commerce, private services)

(d) Non-profit organisations (charities, foundations)

(e) Civil society groups (citizens and informal groups)

(f) Knowledge cluster (universities, research centres, R\&D departments of public and private organisations, knowledge professionals).

- The city as system of infrastructures, services and activities as Context of the solution

- The production and use of small, medium and big data in the city as Object of the solution

The boundaries of the intervention space are set by considering the mutual relationships among these three nodes, see Figure 2.

- The connection between Users (city stakeholders) and Object (city data) had been explored in terms of relationships between city components and classes of local stakeholders related to their role in the city data ecosystem as:

(a) Data collectors, gathering data in the context

(b) Data processors, analysing and structuring data

(c) Data providers, managing the distribution of data

(d) Data consumers, using data

(e) Data subjects, actively or passively providing data on their activities

- The connection between Users (city stakeholders) and Context (city activities) had been examined by taking into account different types of applications and uses of data in supporting strategic decisions, management choices or resource allocation operations.

- The connection between Object (city data) and Context (city activities) had been investigated in terms of structures and mechanisms to align the production and use of data to information needs related to infrastructures, services and local activities.

In synthesis, the problem of the city data governance can be theoretically reframed as an interaction problem between city stakeholders and data in local activities. Therefore, also ill-defined issues such as data manipulation or malevolent uses of data can be repositioned in this new frame to inform the formulation of an appropriate solution addressing the causes of the issues. 


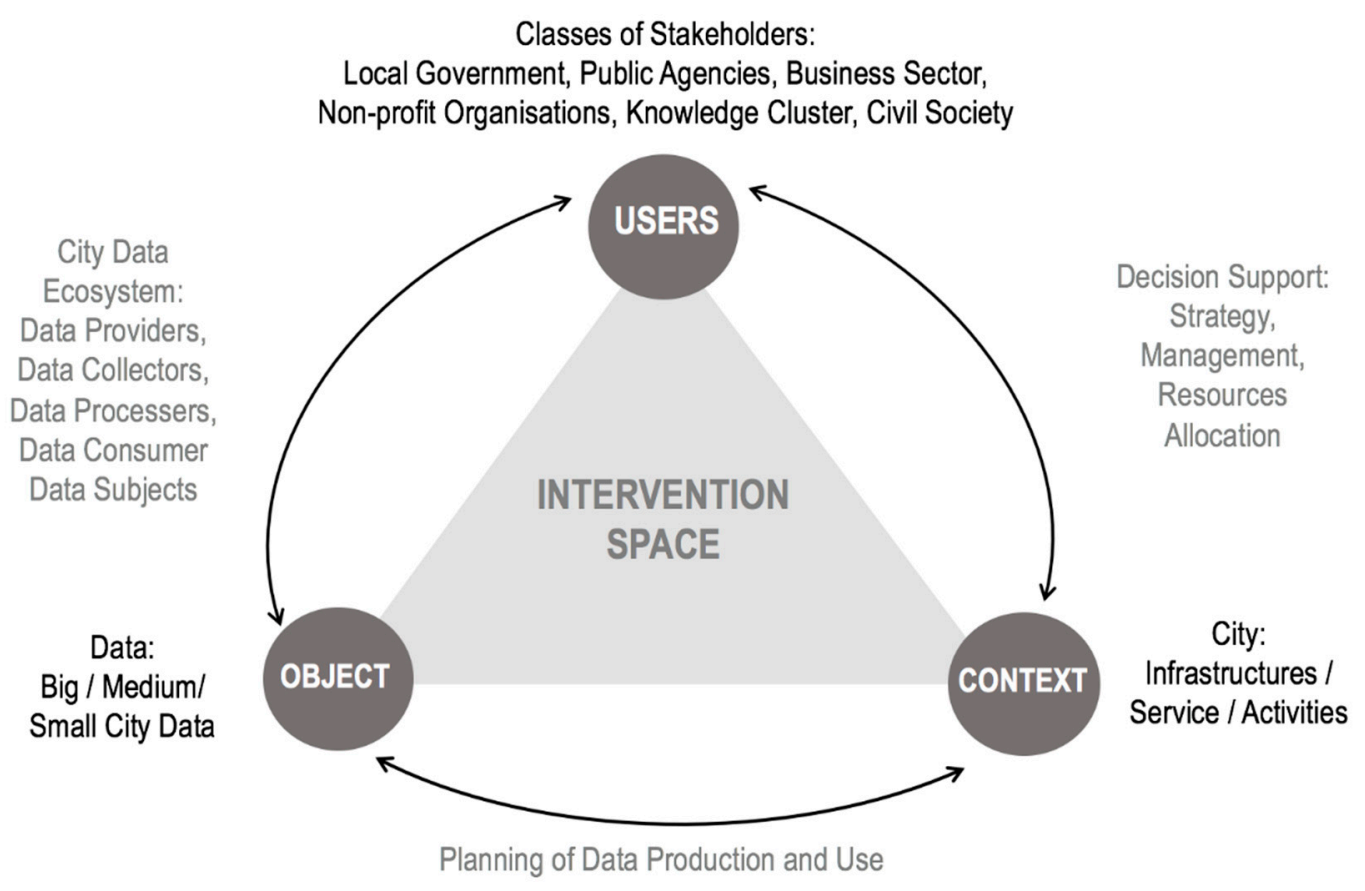

Figure 2. Framing of the problem of the city data governance for defining the intervention space for new solutions to the current issues related to the production and use of city data (source: author's elaboration).

The design research process continued with the identification of two types of solutions, specifically policy instruments, that address problems with structure and constraints comparable with the governance of city data. The definition of new solutions to new problems is indeed often based on studying and repurposing solutions that proved to be effective in addressing similar problems. In this case, the identified policy instruments with these characteristics are urban plans and corporate data governance plans.

Urban plans are traditionally meant to regulate the different interests of local stakeholders in the access and use of urban resources in order to orient an economic, environmental and socially sustainable development at the city level. Data produced in urban environments or associated with city services are nowadays essential resources for city activities, but their management is not yet incorporated in urban planning operations.

On the other side, corporate data governance plans are well-established policy instruments for addressing all the common issues and concerns associated with the management of data in the closed setting of one organisation. However, corporate governance plans are usually limited to a small set of stakeholders on a limited set of operations. Differently, in the city context, the number of stakeholders and the operations they can perform on data are unbounded.

As regarding the three focal points of the intervention space, it is possible to highlight that urban plans are the results of the interactions between Users and Context, local stakeholders and urban services or infrastructures. Corporate data governance plans are focused instead on the relationship between Object and Users, or rather data and stakeholders. These two policy instruments cover therefore complementary functions in outlining a solution aimed to bridge Object and Context, city data and local activities, by taking into accounts the needs, capacities and expectations of local stakeholders, see Figure 3.

The third step of the followed approach consisted in studying the analogies of the types of problems addressed by corporate governance plans and urban plans respect to the problem of the city data governance. The analysis concerned the characteristics, logics, mechanisms included in these types of policy instruments to address the challenges of different goals, actors, institutional structures, operational contexts. In particular, the analysis focused on their commonalities and differences, as well as on identifying the limitations for transferring specific schema and logics to a possible solution for 
the city data governance. The sources for the analysis are constituted by the literature formalising principles and properties of urban plans and corporate data governance plans, but also from the practice and examination of examples of these types of socio-technological tools.

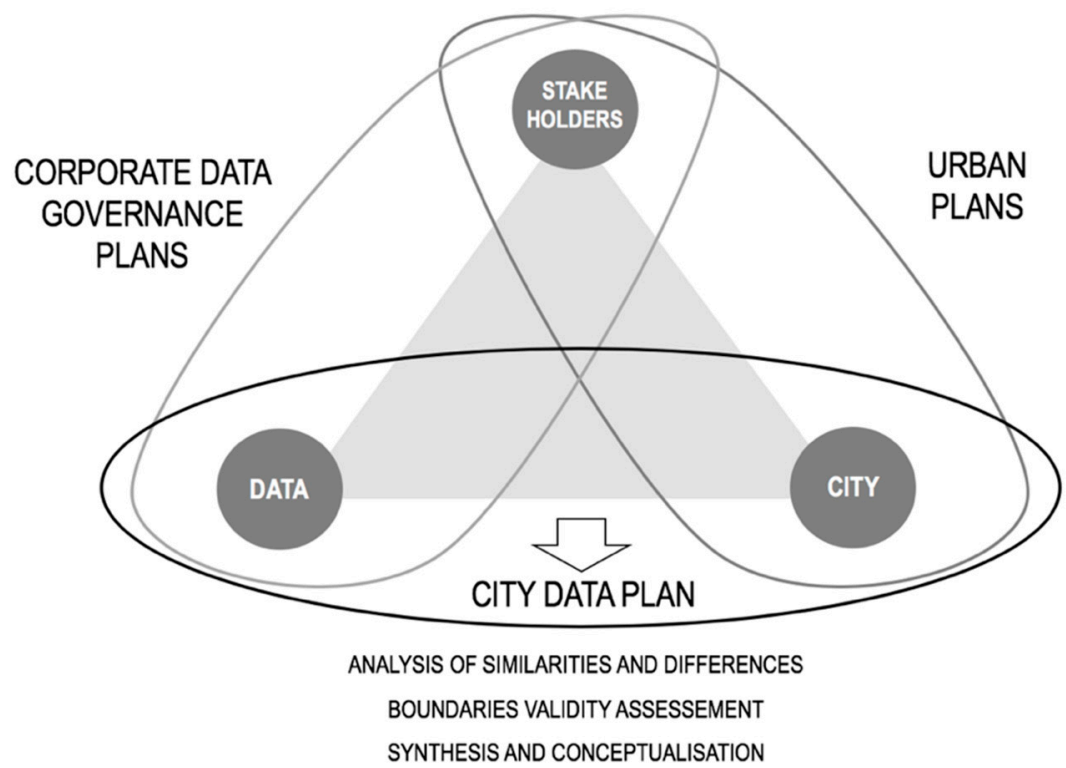

Figure 3. Schema of the rationale for conceptualising the City Data Plan in relation to corporate governance plans and urban plans (source: author's elaboration).

The conceptualisation of the City Data Plan had been drafted throughout this analytic and generative process as synthesis of the instances addressed by urban plans and corporate data governance plans in a new policy instrument (see Section 4). The concept of the City Data Plan (CDP) is therefore the result of a design-oriented reasoning-based process relying on an abductive logic [43]. In other words, the development of the CDP concept as solution meeting all the problem constraints does not result from empirical studies, but from an iterative process of:

- Re-elaboration of the previous knowledge of problems and solutions having characteristics similar to the problem of the city data governance (in this case, corporate data governance plans and urban plans)

- Abstraction of their essential characteristics, such as components and logics

- Generalisation of schema and patterns of the analysed solutions for their transferability to a new problem, the governance of city data in multi-stakeholder context

- Contextualisation of the components and logics of the analysed solutions in a new context and within a new type of policy instrument (see Sections 5 and 6)

The concept of the City Data Plan, as presented in this work, is a theoretical construct designed to outline one possible answer to the two challenges of facilitating the redistribution of the benefits associated with the availability of city data and framing the production and use of city data into a strategy consistent with the development goals of the city and its smart city vision. The intrinsic validity and the limitations of the concept are assessed by confronting the measures included in the CDP and the constraints of the problem of the city data governance (see Section 7).

\section{Developing the Concept of the City Data Plan}

The analysis of the measures commonly established by corporate data governance plans and urban plans is here divided into two sub-sections. Each sub-section is going to report in detail structure, logics, scope and operational aspects of one of the two policy instruments considered in the analysis, and how their characteristics had been readapted to inform the development of the CDP concept. 
The analysis focused on the challenges of extending data governance plans from an enterprise context to an urban context (Section 4.1) and repurposing the mechanisms set up in urban plans to manage the exploitation of urban resources from land use to city data (Section 4.2). Each sub-section is concluded by a synoptic table summarising the key elements discussed in the analytic flow of abstraction and refactoring of the two policy instruments used as basis for the CDP.

\subsection{From Corporate Data Governance Plans to the City Data Plan}

Data governance plans are framed in an enterprise context as the set of measures, organisational arrangements and tools to establish roles and responsibilities in decision-making processes that concern the corpus of data produced by a specific business [44-46]. Corporate data governance plans indicate "who can take what actions with what information, and when, under what circumstances, using what methods" [44]. This definition highlights how the design of a data governance plan concerns more organisational design than technology in itself [47], and in particular the design of the relationships between people, technologies and processes related to data [48].

Data governance and Information technology (IT) governance are indeed two separate dimensions in the business sector. The governance of IT systems concerns the management of IT resources (hardware, software, databases) belonging to the enterprise, and therefore considered as internal resources under the exclusive competence and responsibility of the company owning those assets. Differently, the governance of data requires to take into account the system of relationships between data providers and data users that generate the values associated with data, their respective responsibilities and accountability levels, their agreed standards and metrics for assessing data operations [45,48-50]. Therefore, the main goal of a corporate data governance plan is ensuring clear, not conflictual, repeatable, transparent processes for the management of data among the stakeholders interacting with a specific company and its data [44].

The distinction between IT governance and data governance is currently not clearly established in smart city initiatives. In this domain, the agreements between technology providers and public authorities acting as their direct clients usually bundle together the provision of IT resources (e.g., infrastructures, devices, sensors, data elaboration software), the collection and elaboration of data, and the access to data-driven services provided by the same company. This type of arrangements, still predominant in the offer of the major smart city technology providers such as IBM, Cisco or Samsung, determines a dependence relationship between public authorities and IT companies for the implementation of smart city programmes. This contractual schema inhibits the exploitation of the same IT infrastructure for multiple purposes by stakeholders other than the two parties of municipalities and providers, despite the capabilities of the available technologies. Moreover, the production and use of data is managed and intermediated by technology providers in ways that not necessarily are legible or understandable to their clients or the public. The exclusion of third parties from entering in the process of "smartening" the city generates social conflicts and a growing opposition to smart city initiatives.

The case of Sidewalk Toronto, the smart neighbourhood developed by Sidewalk (one of the Google Alphabet companies), is an example of a smart city initiative strongly contested specifically because the governance of IT infrastructures placed by the company and the governance of data collected in the area by tracing citizens' activities are not clearly distinguished and independently managed [51]. This case points out how the agreements applied to regulate contractual relationships between ICT companies (responsible for the implementation of smart city projects) and public administrations (accountable for decisions on the city development) cannot be simply extended also to the produced data whose use impacts on an extended cohort of stakeholders (e.g., citizens, local businesses, public agencies).

Transferring the distinction between IT governance and data governance from a closed enterprise context to the context of smart city initiatives implies to disentangle the supply of IT resources, the operations on data (collection, processing, application) enabled by the provided IT resources, and the development of services driven by data. The supply of IT resources can be managed independently from data governance arrangements, by revising current contractual schemas between technology 
providers and direct clients. Differently, operations and services centred on data require to open decisions, actions and opportunities to other stakeholders operating at a city scale in a transparent, flexible and inclusive way.

The multiplication of contracts between all the interested parties to city data is not a viable solution in a context in which needs, goals, technologies rapidly evolve. A data governance plan at a city level can be instead a light-weight solution for setting up basic rules, rights and operational plan concerning the production, access, use of city data. On this ground, a City Data Plan (CDP) is configured as a policy instrument aimed to protect and regulate the needs and interests of city stakeholders respect to the governance of city data, counterbalancing the arrangements related to the governance of smart technologies (see Box 1).

Box 1. Separating IT governance and data governance in a CDP.

Example 1. Traffic detection sectors installed along heavy-traffic roads in the city.

Contractual arrangements overlapping IT governance and data governance cover and regulate:

1. Installation and maintenance of the technological infrastructure related to sensors

2. Exclusive rights of the technology provider and its sub-contractors to operate on data

3. The supply of data-related services to the direct client signing the agreements

4. The right to extend access to data to authorised entities

The infrastructure of traffic sensors cannot be reused by third parties or the client for other purposes not included in the contract, the access to data is restricted, the opportunity to generate services based on data by third parties is not usually provided. As a consequence, replication of infrastructures with similar scope under distinct providers and clients is not infrequent. On the contrary, the reuse of data by technology providers remain untraced.

Contractual arrangements separating the installation and maintenance of the IT infrastructure from the use of the infrastructure allow third parties to produce and use data generated from a shared infrastructure of public utility to develop independent services. This type of arrangements is essential to fully exploit the city IT infrastructures flexibly and dynamically, supporting synergies among public and private services, not necessarily bounded to technology providers.

A City Data Plan specifically norms the legitimate uses of shared IT infrastructures taking into account the involved parties interested in data generation and data-related services.

Clarified the scope of a corporate data governance plan and how it can be extended to urban settings, the second step is analysing the essential needs met by this type of policy instruments in the two different contexts of the business sector and city institutions.

According to the literature $[44,49,52]$, the needs driving the elaboration of a data governance plan in an enterprise are:

(a) Increasing revenues and values of data seen as company assets

(b) Containing the costs associated with the growing complexity of data management processes

(c) Addressing communications issues with external stakeholders

(d) Reducing risks and problems related to security, privacy, compliance with established regulations

The critical needs that can be faced by a City Data Plan have a similar rationale but requires additional specifications.

In the city context, as highlighted in the distinction between IT governance and data governance, the central issue is that data produced in urban environments or tracing the activities of people and organisations in the city cannot be considered as assets exclusively owned by the company responsible for their collection or by their direct clients. The lack of clarity between the rights of the "data subjects" and the owners of data is one of the sources of conflicts on city data. On the other hand, new trends in civil society call for recognising data as public resources and therefore expected to generate value for the society at large [53]. In particular, city data are expected to facilitate and improve the provision of public services, but also structurally supporting local businesses and communities in better managing their activities, especially the ones directly impacting on the liveability and growth of cities. 
In this context, a City Data Plan responds to the need of taking into account not only the ownership of the means for the production of data, but also the impact on data on city stakeholders. Therefore, it makes explicit the strategies to maximise the value of data produced in the city for those stakeholders within the specific constraints due to the forms of data ownerships and the related "spectrum" of data licenses (open data, public data, restricted data, private data) [54].

The second need addressed by a City Data Plan concerns the reduction of the extra-costs associated with a disorganised management of city data. For instance, these extra-costs are linked to the replication of efforts for data collection and processing by public and private organisations. The replication of efforts is generally due to the lack of knowledge of data already available, lack of exchange and cooperation mechanisms between data providers, blocks and prohibitions imposed by supply contracts. Other extra-costs are associated with the fragmentation of information, the difficult reuse of information, and the rapid obsolescence of data (see Box 2).

The reduction of part of these extra-costs can be achieved by mapping the status of city data (e.g., available, in progress, planned collection, collected but not processed, archived) in the City Data Plan. This mapping, collectively executed by the organisations involved in preparing the CDP, is intended to survey data resources and data needs (missing data or data to be produced in the future) at the same time. The mapping will also increase the chances of timely finding the required data for specific activities and the value and impact for the organisations producing and using these data, both for public and commercial purposes. In this regard, the third need addressed by a City Data Plan is providing to data producers and user the opportunities of planning their data-driven activities on the basis of the "big picture" of the available data and the remaining to be produced to fulfil emerging needs.

Lastly, a City Data Plan is a relational tool aimed to overcome rigid organisational and technological systems preventing codified cooperative mechanisms to reduce risks and problems related to security, privacy, normative compliance of data-centred actions.

Box 2. Extra-costs due to replication efforts in data collection and processing.

Example 2. Data on railways station users.

The company or public agency owning or managing a railway station usually collect data on people using the spaces or services of the station (e.g., through cameras, sensors, ticketing systems). Police departments or security agencies promote the collection of other data on the premises of the station, in public spaces or parking areas. Part of these data are replicated, as well as their processing. However, without any knowledge of what data are already collected in a specific area, each organisation interested to have information on the same topic (such as the users of the railways station) is forced to operate by supposing that no data are available. Potential complementarities would make more effective and efficient data-related operations.

Moving to the building blocks of a data governance plan, its components established in an enterprise context are [47]:

(1) Dedicated organisational bodies composed of representatives of both the company and the relevant stakeholders expected to establish collective goals, a shared decision-making framework and responsibility assignments

(2) Well-defined standards and processes involving data

(3) A set of technologies to support these processes, not necessarily self-included in the systems for data production and management.

In the following, these three essential components of corporate data governance plans are analysed in relation to the concept of the City Data Plan.

The organisational bodies proposed as institutions involved in the preparation and management of the City Data Plan are modelled on the basis of those defined by Kitchin in his work on "the Programmable City" [24] and already discussed in Section 2. The variant proposed here consists of the following institutional bodies: 
- A multi-stakeholder advisory board operating at the urban level

- A cross-sectorial teams of domain experts and data experts, affiliated with and representing the different data providers in the city data ecosystems, instead of a team of data experts internal to the public administration

- A coordination team to support the communication and cooperative operations among the different technical/not technical teams of data providers and data users, instead of a centralised technical team within the public administration

- A team specialised in direct emergency response (for severe crisis) and coordination of emergency response across the technical teams associated with the plan for low-level risks, instead of one team for a centralised emergency response team.

The main difference between the structures proposed by Kitchin and the ones associated with the CDP concerns the importance of mixing domain experts and data experts. Their combination is indeed essential to make informed judgements and decisions on the structure of city data protocols taking into account real operational needs due to the nature of services and activities. The second difference is proposing distributed institutional structures to minimise or avoid functional changes or restructuration within each organisation involved in the plan, preferring instead flexible mechanisms to differentiate the level of engagement compatibly with capacity and scope of each local actor. The last difference consists in outlining multiple teams within distinct organisations coordinating themselves with public authorities but not necessarily dependent on their capacity, availability, willingness to support multi-level and multi-actor processes of collaboration for data production and use (see Box 3).

Box 3. Distributed structures for city data governance.

Example 3. Data covering the educational offer at the urban level.

Representatives of the major educational institutions of the city can be involved in the advisory board of the CDP to bring the instances of education professionals, students, and families.

Each school, public and private, can internally identify one referent for the CDP, available to collaborate in a mixed team of domain experts (in this case on education) and data experts covering a specific area of the city in the aggregation, collection, production, analysis of data on education-related topics. Teams working in different areas on the topic of education, or working on different topics but in the same area, are facilitated in their interactions by the coordination team. The coordination team support the cooperation of distributed team in joint initiatives to achieve specific goals set by the advisory board.

Indeed, public policy development is under the competence of public authorities and city plans are regularly prepared under the initiative of local governments in compliance with regional and national laws. However, considering the lack of specific laws and regulations on the governance of city data, the initiative of developing policy instruments on this topic can also be promoted by other actors operating in the city. If they have the public influence to aggregate the necessary consensus on this type of initiative (for instance, universities, private foundations, structured civil society organisations), they can progressively involve other public authorities and the business sector. A proto-example of this type of spontaneous processes aspiring to a subsequent institutionalisation is the "TADA Manifesto" [9], mentioned in Section 1. Nevertheless, the engagement of municipalities and local governments is essential to mediate and orchestrate the plurality of interests and actors at the local level in compliance with the legal framework.

Other aspects characterising the organisational bodies associated with corporate data governance plans are:

- The definition of their organisational goals

- The position of the decision-making power (defined as "locus of control")

- The roles and responsibilities covered by each person implementing the plan [45].

The organisational goals are classified in the literature as formal or functional. Formal goals are oriented to maintain or increase the value of data as company asset (by measuring or assessing the 
effectiveness of the data governance respect to the business goals). Functional goals orient instead concrete decisions on data management 46].

The decision-making power is usually classified respect to its position in the organisation (centralised or decentralised) and the nature of the relationships between decision-making authorities (hierarchical or cooperative) [50,52].

The responsibility assignments distinguish the different roles of people involved in the data governance in:

(a) Responsible for the execution of activities

(b) Accountable for the activity and therefore making and authorising decisions

(c) Consulted to provide inputs and support before that a decision is taken

(d) Informed of decisions and their outputs [50]

Usually, the responsibilities established within a corporate data governance plan are oriented to mitigate the "dysfunctional aspects" of an organisation [46]. Therefore, their schema does not necessarily replicate internal hierarchies and divisions that can be affected by a mismatch between formal protocols and actual operational procedures.

Having considered these aspects for conceptualising a City Data Plan, it is important to highlight the following points.

The formal goals of a City Data Plan need to be explicitly defined at a political level. They emerge through the concertation with other local stakeholders in the advisor board in order to construct a shared vision of what type of data as public assets can generate value, who benefit of this value and in what forms. Indeed, as mentioned in Section 2, the hidden political choices driving city data operations (what data are collected, where, why, by whom, for what purpose) are one of the most problematic aspects in the governance of city data, facilitating data manipulation and malevolent uses of data. Making explicit the political assumptions and directions for the future of a city in relation to a part of its assets represented by city data is an important step to set the boundaries of the public arena in which the different stakeholders operate.

The functional goals of a City Data Plan require instead to be set at three levels (see Box 4):

(1) Within the single organisation participating in the city data ecosystem in the capacity of data producer or data user

(2) Among the subsets of organisations implementing interdependent data-driven or data-dependent activities

(3) Across different subsets of organisations covering the same domains or running similar operations to establish minimum standards and common operational principles

Decision-making powers are necessarily decentralised and cooperative at the urban level to implement the measures for achieving the established formal goals and facilitating cross-organisational exchanges. On the contrary, at the level of the single organisation, they can be arranged in forms (centralised or not, hierarchical or horizontal) appropriate to their internal structures, by keeping stable a core of referents to enable the work of the coordination team.

At the urban scale and in open multi-stakeholder partnerships, the responsibility assignment is set at the level of organisations and their subunits. Within each organisation, that roles can be redistributed in terms of tasks to specific individuals, and dynamically readapted and readjusted to contingencies, without affecting inter-organisational arrangements. 
Box 4. Formal and Functional goals in the CDP.

Example 4. Mobility and elderly population.

The formal goal concerning the data production and use on the topic of mobility in the city is established by the representatives of local stakeholders involved in the advisory board. This formal goal is an expression of political orientations and a shared vision for the city. For instance, a formal goal could be facilitating the access to public transportation for the elderly population in the south of the city.

Distinct functional goals are established at the level of the single organisation involved in the strategy to reach this goal. For instance, local transportation companies can decide to separate data on over 65 years old from the remaining age bands of their users by revising their ticketing systems to exchange these data with the mobility department of the local council.

Other functional goals can be collectively set among organisations that benefit from similar type of data because they provide similar types of services (e.g., local bus companies and taxi drivers) or implement inter-connected activities (e.g., mobility and social care department of the local council, homecare professional associations, and bus companies).

The City Data Plan is intended to trace the formal and collective goals, by area, domain and organisation, specifying the role of each organisation in the implementation of a specific strand of the plan.

The second building block of a corporate data governance plan consists of standards and processes on data. The decision protocols in this policy instrument conventionally cover five domains: uses of data, appropriateness of data to the intended uses, nature of the associated metadata, access requirements of data, and data lifecycle (planning, production, management, retirement) [49]. These decisions protocols are focused on data quality, privacy protection, security, normative compliance, integration among different systems and data sources, definition of the data-related services to be provided [44]. More in general, data governance decision protocols include the entire set of rules to operate with data through the allocation of tasks. They also include the "rules of engagement" of the company staff and other organisations aimed to solve issues and conflicts of competencies and responsibilities between internal and external people to enable efficient "proactive, reactive and on-going data governance processes" [44].

The definition of the decision protocols for a City Data Plan is challenged by the plurality of potential uses of city data for different stakeholders. This plurality implies multiple concurrent standards to assess the quality of data. These standards are not related only to the correctness of data, but to their suitability to be used by local stakeholders in terms of licensing, spatial and temporal granularity, integrability, interoperability. In this context, the quality of data is conditioned by their relevance to inform individual, organisational and collective decisions. Therefore, high-quality data are the ones providing specific inputs to the ordinary and extraordinary activities concurring to city development (e.g., launching a new service or a community initiative, supporting new measures for the reduction of energy consumptions in commercial buildings, making more efficient the parking slots allocation in the city centre, and so on). As will be examined later, high-quality data do not necessarily involve the collection and processing of big data, or real-time information, or low granularity focus, but a specific tailoring on the type of decisions they are intended to support.

A data governance strategy, at a corporate level but also at the urban level, is given by the matrix connecting the decision protocols to the responsibility assignments of the organisational structures involved in the process [49].

The third building block of a corporate data governance plan is constituted by information and communication technologies able to support the dynamic alignment between data management processes and the goals set by the data governance plan. These technologies can be software dedicated only to the management of the plan and reserved to the organisational bodies in charge for its execution. More frequently, the implementation of a corporate data governance plan is supported by a generalist tools for team management such as shared repositories, alerts, mailboxes, and so on.

The implementation of a City Data Plan requires technologies easily appropriable by different units and organisations, with different levels of data expertise and domain knowledge of city services and activities. These technologies are also expected to be flexible and reactive to needs and constraints dynamically evolving, as well as the variable number and composition of the stakeholders involved in the plan. Lastly, differently from the corporate context, spatial and temporal coordination in the production, processing and 
use of data is the key challenges. Indeed, different actors can operate at different scales (e.g., city district and neighbourhood) on the same types of data, or in different areas and periods on complementary data. In both cases, effective coordination can optimise their efforts and resources, facilitating the reuse of available data resources or infrastructures. In the next section, the type of tools usually adopted to communicate and manage urban plans updates are suggested to partially fulfil those requirements.

The following Table 1 summarises the various aspects of corporate governance plans reinterpreted in the conceptualisation of the City Data Plan and analysed in this section.

Table 1. Overview of the key aspects of corporate data governance plans informing the conceptualisation of the City Data Plan (source: author's elaboration).

\begin{tabular}{|c|c|c|}
\hline & Corporate Data Governance Plan & City Data Plan \\
\hline Goal & \multicolumn{2}{|c|}{ Design of the relationship between people, technologies and processes related to data } \\
\hline Focus & IT assets $\neq$ stakeholders ${ }^{\prime}$ interests & $\begin{array}{l}\text { Ownership of smart technologies } \neq \\
\text { ownership or rights on data }\end{array}$ \\
\hline Needs & $\begin{array}{l}\text { (a) increasing revenues } \\
\text { (b) containing costs } \\
\text { (c) reducing risks } \\
\text { (d) improve communication }\end{array}$ & $\begin{array}{l}\text { (a) increasing value of data } \\
\text { (b) containing data mismanagement } \\
\text { extra-costs } \\
\text { (c) overcoming organisational barriers } \\
\text { (d) enabling coordinated data-centred } \\
\text { actions }\end{array}$ \\
\hline Organisational bodies & $\begin{array}{l}\text { Internal teams and coordination units with } \\
\text { external stakeholders }\end{array}$ & $\begin{array}{l}\text { (1) multi-stakeholder advisor board } \\
\text { (2) cross-sectorial multi-team of domain } \\
\text { experts and data experts } \\
\text { (3) coordination team } \\
\text { (4) emergency response team }\end{array}$ \\
\hline Organisational goals & $\begin{array}{l}\text { Formal goals: } \\
\text { Business oriented } \\
\text { Functional goals: } \\
\text { Operational }\end{array}$ & $\begin{array}{l}\text { Formal goals: political } \\
3 \text { level of Functional goals: } \\
\text { - } \quad \text { Within single organisations } \\
\text { - } \quad \text { Among interdependent organisations } \\
\text { - } \quad \text { Among organisation operating in the } \\
\text { same domain or area }\end{array}$ \\
\hline Decision-making power & $\begin{array}{l}\text { Position of power: } \\
\text { centralised or decentralised } \\
\text { Relationships in decision making: } \\
\text { hierarchical or cooperative }\end{array}$ & $\begin{array}{l}\text { Decentralised and cooperative at the urban } \\
\text { level } \\
\text { Flexibility on organisational arrangements } \\
\text { (centralised or decentralised, hierarchical } \\
\text { or cooperative) }\end{array}$ \\
\hline Responsibility assignment & $\begin{array}{l}\text { Roles of individuals: } \\
\text { - } \quad \text { Accountable for decisions } \\
\text { - } \quad \text { Responsible for action } \\
\text { - } \quad \text { Consulted for inputs } \\
\text { - } \quad \text { Informed of decisions }\end{array}$ & $\begin{array}{l}\text { Roles appointed to organisations } \\
\text { Internally distributed to individuals }\end{array}$ \\
\hline Themes of decision & $\begin{array}{l}\text { Uses of data } \\
\text { Quality of data } \\
\text { Metadata } \\
\text { Access requirements of data } \\
\text { Data lifecycle }\end{array}$ & $\begin{array}{l}\text { Plurality of uses of data } \\
\text { Relevance of data } \\
\text { Appropriateness of data } \\
\text { Legibility of data } \\
\text { Licensing } \\
\text { Transformability and reuse }\end{array}$ \\
\hline Focus of decision protocols & $\begin{array}{l}\text { Data quality } \\
\text { Privacy protection } \\
\text { Security } \\
\text { Normative compliance } \\
\text { Interoperability } \\
\text { Integrability }\end{array}$ & $\begin{array}{l}\text { In addition: } \\
\text { Rules of engagement at urban and } \\
\text { inter-organisational level } \\
\text { Conflict-resolution procedures }\end{array}$ \\
\hline Technologies & $\begin{array}{l}\text { Technologies monitoring the data managen } \\
\text { Technologies supporting spatial and tempo }\end{array}$ & $\begin{array}{l}\text { ent processes } \\
\text { al coordination of the involved stakeholders }\end{array}$ \\
\hline
\end{tabular}




\subsection{From Urban Plans to the City Data Plan}

Urban plans are legal instruments codifying actions and interactions between public authorities and society for the formulation, implementation and evaluation of spatial planning activities. In this sense, they can be defined as "institutional technologies" [55], complex mechanisms to frame the operational rules for urban transformations driven by different types of institutions. In the Netherlands, France, Italy, Spain and other European countries, urban plans are municipal laws associated with visual models of the contents of the law (the actual City Plan). The implementation of plans is enforced at the local level, but connected to regional and national laws and regulations. In the UK, urban plans are not laws [56], but rather policy instruments to inform decisions about the future permitted developments [57].

Under both types of frames, the elaboration of urban plans requires a priori extensive negotiation and dialogue among city stakeholders and authorities charged of planning competencies and responsibilities. Indeed, the central goal of urban planning is managing the coexistence of different instances and interests in a shared space [58] and defining a vision for the future of the city based on what is possible, not what is probable [59]. To get closer to that vision, urban plans includes lists of actions, rules for actions, targets and strategies to frame collective, independent and unpredictable activities implemented by different stakeholders [60]. The tensions between vision and operational aspects, normativity and adaptability to future settings, high-level coherence and low-level self organisations make urban plans capable of addressing the issues of city data governance in their complexity by outlining manageable solutions.

As introduced at the beginning of this section, the conceptualisation of the City Data Plan had been developed by taking into account the practices of making urban plans, but reasoning on how to readapt these practices from the management of land use to the management of data as urban resources.

Urban plans are indeed traditionally aimed at regulating land uses and the production or transformation of the built environment at the urban and local scale. As a result, the main domains covered by urban plans are housing and industry districts spatial developments, public and private services localisation, urban rehabilitation programmes, mobility, infrastructures management and expansion, green areas and cultural heritage protection, and other related themes. In every case, land use regulation is used as spatial proxy for the implementation of a future social and economic vision of the city [19]. This vision is always oriented by political considerations, but relies on specific strategies and operative measures to create and redistribute the value generated by the desired urban transformations. While in traditional urban plans the desired urban transformations are linked to the management of land use conversions, in a City Data Plan the desired horizon for the governance or city data (defined as "formal goal" in corporate data governance plans) is associated with progressive transformations in the competencies and responsibilities of local stakeholders in the production and use of different types of data. The shared vision at a political level is operationalisable by setting specific targets concerning transfer mechanisms and balancing of competencies and responsibilities on city data.

The non-organised management of the production and use of data in the city by public and private organisations is partially attributable to the lack of a long-term vision scaffolding contingent smart city initiatives, technology provision contracts, creation of ICT infrastructures, experimentation of new devices and data collection methods in urban environments. Reframing these types of operations within a coherent picture oriented by shared explicit goals for the future of the city is a measure to rationalise also public and private investments in data production, acquisition and use. Moreover, outlining the grid of targets, competencies and responsibilities in the city data ecosystem, as well as rights and benefits of shared data governance mechanisms, is the precondition for enabling city organisation to participate in actively contributing to implement the long-term vision of a data-enhanced city development.

The most critical aspects in the conceptualisation of the City Data Plan is that the equivalent of land in urban plans are not data, but the organisations contributing to the city data ecosystem. 
The potential value of land in a city is directly connected to its use or destination. Similarly, the value generated from city data is directly linked to the allocated work-time of the limited human resources of local organisations that can be devoted to data-related operations. Data, differently from land, are not meant for undivided uses and have a high yield because they can be potentially reused and recombined an indefinite number of times by un undefined number of users. Nevertheless, their management, maintenance and users' support operations are time-consuming intensive activities impacting on the capacity of organisations participating in the city data ecosystem. Local organisations can be included in the city data ecosystem in the roles of data collectors, data producers, data processors, data users, data communicators, and data subjects. These roles can dynamically evolve, but taking into account that the generation of social and economic value from city data is a concurrent process with the generation of value from the core activities of local organisations.

The preliminary assessment of the "extra-workload" for each organisation contributing to the cooperative governance is an essential element for defining the policies included in the City Data Plan. For instance, these policies can include managing access and forms of support provided for specific types of data, and the measures to contrast negative externalities due to several requests exciding the capabilities of local organisations human resources. The assessment of the extra-workload corresponds, for instance, to the common practice in urban planning activities of assessing in advance the extra-traffic load due to the realisation of new mobility infrastructures in already congested urban areas. It is simply based on defining the capacity of an infrastructure and the volume of expected users. Coming back to organisations, the assessment of the extra-workload is given by the role of the organisation, the type of data provided into the city data ecosystem, the working hours potentially dedicated by the available human resources in the coordination and joint operational activities related to the City Data Plan.

The parallelism between land use and data-related workload of local organisations can be defined more in detail considering on one side the land use classification, and on the other side the different type of workloads associated with different types of data. In both cases, a macro-level classification help in distinguishing the type of required infrastructures, resources, and investments to implement the actions in the plan.

As regards the land use, specifically for the built environment, a distinction is usually made between residential areas, areas reserved to public services, and commercial areas. Residential areas are usually subjected to slow transformations. The value of each residential unit is high for those living or owning that unit, but not for others. Nevertheless, the aggregation of residential units in blocks or neighbourhood became a matter of public interests in planning future developments and in case of collective problems. Commercial areas are the ones with the highest profitability and the fastest rate of transformations. They require intense investments and infrastructures to fully exploit their potential. In between, areas reserved to public services (e.g., hospitals, schools, cultural and sports buildings, parks, station) are essential for the functioning of the city, and their value is not reducible to their profitability.

Similarly, different types of data determine distinct sets of resources impacting on the workloads of local organisations. In this case, a macro-level classification to structure the measures of the City Data Plan can consider the differences associated with big, small and medium data. Big data (as well as commercial areas in land use classifications) have the highest profitability, but requires investments, specialised infrastructures for their collection, highly skilled workforce and resources for their processing and exploitation. Moreover, their production is limited to a few organisations, usually specialised on data management and dealing with data as core business. On the opposite side, small data such as project reports and local surveys usually contain static structured information on the context, but do not have a direct commercial value and not necessarily require specialised infrastructures or data experts for their collection. The production of small data is virtually extended to every organisation working in the city as side operation oriented to monitor and document their own activities. Even though the value of these small data is higher for their producers than for third parties, 
their reuse can inform decision-making processes and complement analysis based on other sources. Therefore, their aggregation at the city level is a matter of public interest (see Box 5). In between, medium data generate by public administration, universities, cultural institutions to fulfil part of their organisational missions (e.g., government transparency or knowledge generation) have a social value in themselves but could be instrumental in complementing and bridging both small and big data sources.

In the framework of a cooperative city data governance instantiated in the preparation of the City Data Plan, different types of data producers are called to make public what type of information they can provide, in which forms, under what conditions. As mentioned before, this mapping can reduce replication efforts of the same data sources, facilitate matchmaking between data producers and other actors in the city data ecosystem making legible all their reciprocal roles and interests.

Box 5. Value of aggregated Small Data.

Example 5. Community engagement.

The reports of the engagement activities carried out by local non-profit organisations in the last ten years are a significant example of small data. The value of each single report is marginal for actors external to the organisation that produced them.

However, the lack or low accessibility of this type of documents (rarely upload on websites) from the same organisation or from different entities working on the same communities can generate issues of public interest. The efforts to support local projects and initiatives with public or private funds remain untraced. Additionally, it is impossible learning from past experiences to inform future initiatives, overcoming barriers that are already known, or leveraging on resources and themes that resulted successful. Besides, the lack of any record of engagement processes can result in distrust toward non-profit organisations, donors, and public authorities usually considered responsible or accountable for community well-being and public participation processes. On the contrary, their aggregation can clearly depict local trends and critical issues of each community helping to strategize future interventions.

Sharing this type of documents in a common repository accessible to the CDP institutions involves minimal work for local charities and civil society groups, but enhance their role in the city data ecosystem as data providers.

A CDP maps also this class of data producers, their working area and their domain of activity, making easier their active involvement with other data providers and users.

Considering the type of decision framework characterising urban plans, it is important to notice that urban plans are in general articulated as multi-level policies, incorporating high-level strategies for the development of the city at the urban level and more detailed plans for local interventions. These detailed plans can also be prepared independently from the general plan but within the same grid of rules and actions. This articulation relaxes the burden of taking overall and detailed decisions at the beginning of the plan-making process and allows general plans to be flexible enough for addressing local changes, incorporating new needs, and taking advantage of new opportunities.

Analogously, a City Data Plan is intended to be used as a general framework to organically accommodate lower-scale and contingent arrangements between data producers and users determined by specific smart city programmes, local projects, area-based initiatives. While the City Data Plan outline the grid of norms and protocols regulating the data production and use in the city, lower-scale arrangements will define roles, activities, values created in a specific data-related process (see Box 6).

In contemporary urban plans, the grid of norms framing local interventions pursue the goal of ensuring a good functional mix between different land uses (commercial, industry, residential, public services, green zones) at every scale, from neighbourhoods to urban areas. The structure of the desired functional mix results from the assessment of the existing resources and the established vision for the future of the city. In a City Data Plan, this grid of norms and protocols concerns specifically the articulation of partnerships and mutual relationships among the plurality of organisations in the city data ecosystem. In particular, the City Data Plan outlines relational schemas among organisations contributing to the same chain of production and use of data, or operating in the same territory, and therefore collecting and using data about the same services, infrastructures, communities. The overall 
goal is ensuring a balanced ecosystemic functioning of the different actors aimed to minimise conflicts and maximise the value creation for single organisations and the society at large.

The elaboration a City Data Plan enables to strategize and manage the appropriate operations and infrastructure for the goals to pursue in terms of data-driven city developments at short, medium and long term, but also to effectively segment the efforts in multiple sectorial sub-plans covering specific types of data or areas.

Box 6. Multiple timeframes for the general CDP and its sub-plans.

Example 6. Assessment of the environmental quality at the urban level.

The CDP maps data sources and organisations working on this topic or potentially contributing to this goal. The information needs can be dynamically updated as the corresponding data sources are acquired, or a new type of data become required by a group of stakeholders.

In parallel, sectorial sub-plans can be put in place by limiting the coverage of data to a specific area or the application of data within a single smart city initiative.

The CDP continue to work as a framework of rules and protocols also for its sub-plans, while they are temporally and operationally independent from the general CDP.

Urban plans are usually prepared by municipal administrations, considered accountable for the decisions about concessions and authorisations for future developments granted to privates. Nevertheless, the implementation of urban plans is the result of a continuous negotiation process between public administration and privates. The standards mechanisms for facilitating and regulating these negotiation processes can be classified in incentives, disincentives and land rights transfers [61]. Incentives and disincentive are aimed to support or limit specific types of transformations (e.g., interventions aimed to increase the population density), while right transfer mechanisms are non-compensatory forms of land trade to achieve goals of public interests (in opposition to coercive monetary forms of land trade such as expropriations).

These three implementation mechanisms can be effectively transferred to the City Data Plan (see also Box 7).

- Compensatory mechanisms should incentivise the production and sharing of data having a public interest and community applications, but not constituting the core business of the organisations able to produce them. The acknowledgement of the public interest and distributed benefits linked to the production of specific data can be related to a redistribution of costs. In this sense, the data featurization proposed by Finch and Tene [38] could become a standard sustainable practice at an organisational level. This practice should be extended not only to major data producers, but also to organisations actively producing data-related services in local communities or companies having the opportunity to collect data of public interest even if that operation is not in their core business.

- Monetary and non-monetary disincentives should be applied to penalise the overproduction and distribution of data generating unsustainable overloads for local infrastructures and organisational resources. Other disincentives can be applied to the production of data having evident discriminatory or malevolent applications previously identified.

- Data rights transfers mechanisms should support the exchange and reciprocal access to data between subsets of organisations that operate in the same area or benefiting from synergic efforts (e.g., by avoiding the replication of data collection or data analysis processes). These rights can be granted temporarily, in line with the needs of the involved organisations, but in compliance with the rules established in the CDP. The principles regulating data rights transfer mechanisms are established by the multi-stakeholder advisory board and operatively managed by the cross-sectorial domain and data experts' teams.

The exchange value of data is analogous to land rents and position rents regulating land uses and trade, and not necessarily impact on the core business of the involved organisations. The exchange 
value of data is assessed on the basis of the type of decisions supported for the involved parties. Indeed, the same type of data can have different values for distinct subjects because they can enable strategic decisions on future actions (high value), resource management decisions on current arrangements (medium value) and decisions on contingent resource allocations (small value).

Box 7. Incentives, Disincentives, Data Rights transfer mechanisms.

Example 7. Incentives.

The public agency in charge of monitoring water supply infrastructures at a city level relies for its operation on in-house predictive models and data sources. However, the agency needs fine-grained qualitative data to assess status and issues for the customers served by the oldest section of the aqueduct. Plumbers operating in the area have direct knowledge of issues and recurrent problems to the hydraulic systems of local buildings. While it is evident that the core business of plumbers is not in data collection or provision, their occasional cooperation in reporting water-related issues can be supported through a system of equitable and sustainable monetary incentives regulated in the CDP. Indeed, ensuring safe water supply services is essential for the collectivity and it can motivate a collectivisation of costs through taxation (with minimal budgets allocated for distributed data collection) or ad hoc arrangements between water supply agency and plumbers associations.

Example 8. Disincentives.

Restrictions on the use of antennas and repeaters within the municipal boundaries for telecommunication companies making profits from tracking their users by using procedures in contrast with the policies included in the CDP. Limitations in the access to municipal concessions can be associated with softer measures such as initiatives to promote companies adhering to data ethics compatible with the principles and goals of the CDP.

Example 9. Data Rights Transfer.

Large retailers are among the few producers of medium data in the city, besides major public authorities and specialised companies. Their data are private and for internal use only. However, a part of their data can serve public interest purposes without causing any commercial damage to the retailers. For instance, aggregated data on buying habits on alcohol vegetables can help in assess specific health risks at the urban level or the actual impact of food education campaigns. The temporary access to those data by public health agencies or local authorities can be possibly exchanged for other information such as the socio-demographic projections of future urban expansion lots. Criteria and constraints for the negotiation of the data rights transfer are established by the advisory board.

Besides the mechanisms described above, the implementation of urban plans is enabled by preliminary operations aimed to prepare settlements becoming functional. These operations include the "primary infrastructuring" of new development areas and their maintenance (e.g., water and waste management infrastructures, electricity, public lightning, roads and local streets) and the "secondary infrastructuring" with the provision of essential public facilities for education and health services. The costs associated with these operations are usually covered by fees required by the subjects authorised to intervene on the built environment and by local taxes.

The implementation of a City Data Plan is based on unavoidable preliminary operations to infrastructure a cooperative governance of city data that include the survey of the information needs and data resources of local organisations. The preliminary operations also include communication and dissemination of the rules of engagement and protocols for the shared governance of city data included in the City Data Plan, and training to organisations not usually considered as data providers (such as community organisations). The "secondary infrastructuring" of a City Data Plan is constituted by data collection, processing and distribution of those data of which only the public sector can have access (e.g., multi-scale census data, but also taxes and income data). This exclusive competence on specific types of data is due to upper-level organisational structures or regional and national laws.

Lastly, the exceptional aspects of urban plans as laws and policy instruments is that they are constituted by two parts: descriptive documents including regulations and policies, and thematic maps used as "visual models" of the law or policy in itself. Maps representing urban plans dispositions, more 
than the descriptive documents, are the most effective communication support among the stakeholders interested by urban transformation. The map representing an urban plan commonly works as media to negotiate local interventions with the public administration or other involved actors by visualising the status of affairs and its projection in the plan.

As mentioned before the main object of a City Data Plan is not the land use but the capacity of local organisations of contributing to the production, management and exploitation of city data. Nevertheless, thematic maps are appropriate media to represent data resources under shared governance. Indeed, city data have always a spatial relationship with the urban environment because they are collected within the city, from urban infrastructures and local services.

A spatial representation of the status of data resources can support a rapid understanding and a real-time assessment of the advancement and implementation of the City Data Plan. A map can highlight areas, scales, timing, and themes that are already covered by existing data sources and the ones to be prepared for achieving the city development goals and the specific smart city vision shared among the involved stakeholders. Moreover, the spatial mapping of data providers and other actors involved in the city data ecosystem can facilitate proximity-based cooperation mechanisms or call for actions to support the consolidation of data on uncovered areas, especially as regarding the collection of local information needs.

In addition, the readiness of digital technologies such as map-based visualisation tools makes the investments specifically related to the technical implementation of a City Data Plan particularly low because no new technologies are needed. Standard Web-GIS tools are already appropriate to the scope of a City Data Plan, even though they could be integrated by more advanced features to support multi-actor coordination and cooperation mechanisms.

The following Table 2 summarises the aspects of urban plans reinterpreted in the conceptualisation of the City Data Plan and analysed in this section.

Table 2. Overview of the key aspects of urban plans informing the conceptualisation of the City Data Plan (source: author's elaboration).

\begin{tabular}{|c|c|c|}
\hline & Urban Plans & City Data Plan \\
\hline Goal & $\begin{array}{l}\text { Regulating the access and exploitation of } \\
\text { urban resources taking into account public } \\
\text { and private interests in the transformation } \\
\text { of the built environment }\end{array}$ & $\begin{array}{l}\text { Regulating the access and exploitation of } \\
\text { city data as a new layer of urban resources } \\
\text { linked to the generation of value in the city }\end{array}$ \\
\hline Scope & $\begin{array}{l}\text { Outlining a vision for the development of } \\
\text { the city by setting a set of desired targets to } \\
\text { reach in a defined temporal frame }\end{array}$ & $\begin{array}{l}\text { Realigning smart city visions and the } \\
\text { output/outcome of data produced by smart } \\
\text { city technologies }\end{array}$ \\
\hline Nature & Municipal law or local policy & Policy instrument \\
\hline Key resource & $\begin{array}{l}\text { Land (organisations as means for the } \\
\text { valorisation of local resources) }\end{array}$ & $\begin{array}{l}\text { Organisations (data as means for enhancing } \\
\text { the full potential of local services and } \\
\text { social capital) }\end{array}$ \\
\hline Risk assessment & $\begin{array}{l}\text { Extra-load of existing infrastructures and } \\
\text { built areas }\end{array}$ & $\begin{array}{l}\text { Extra-workload of organisation involved in } \\
\text { the city data ecosystem }\end{array}$ \\
\hline Classification schema & $\begin{array}{l}\text { Land use classification } \\
\text { - } \quad \text { Commercial (high profitability and } \\
\text { - } \quad \text { Rest transformation) } \\
\text { value for residents) } \\
\text { - } \quad \text { Industrial } \\
\text { - } \quad \text { Services } \\
\text { - } \quad \text { Green areas } \\
\text { Etc. }\end{array}$ & $\begin{array}{l}\text { Classification of engagement /effort by type } \\
\text { of data produced: } \\
\text { - } \quad \text { Big data: high profitability, highly } \\
\text { skilled workforce, investments in } \\
\text { dedicated infrastructures } \\
\text { - Medium data: service-oriented, skilled } \\
\text { workforce, dedicated } \\
\text { soft infrastructures } \\
\text { - Small data: high utility but low } \\
\text { profitability, no } \\
\text { dedicated infrastructures }\end{array}$ \\
\hline Decisions framework & \multicolumn{2}{|c|}{ Multi-level temporally distributed } \\
\hline
\end{tabular}


Table 2. Cont.

\begin{tabular}{|c|c|c|}
\hline & Urban Plans & City Data Plan \\
\hline Competence & 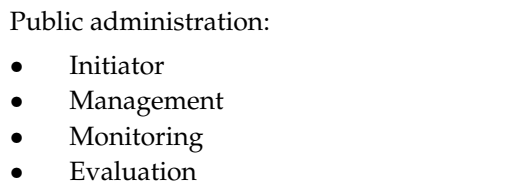 & $\begin{array}{l}\text { - Initiator: public or private entities } \\
\text { having public relevance } \\
\text { Management, Monitoring, Evaluation: } \\
\text { extended to other actors in the city } \\
\text { data ecosystem }\end{array}$ \\
\hline Implementation mechanisms & $\begin{array}{l}\text { - Compensatory mechanisms } \\
\text { and incentives } \\
\text { Monetary and } \\
\text { non-monetary disincentives } \\
\text { - Land Rights Transfer }\end{array}$ & $\begin{array}{l}\text { - Compensatory mechanisms and } \\
\text { incentives to produce socially } \\
\text { relevant data } \\
\text { Monetary and non-monetary } \\
\text { disincentives of data replication efforts } \\
\text { Rights Transfer to support cooperation } \\
\text { in the data production and use }\end{array}$ \\
\hline $\begin{array}{l}\text { Preliminary operations to } \\
\text { implement the Plan }\end{array}$ & $\begin{array}{l}\text { - } \quad \text { Primary infrastructures } \\
\text { - } \quad \text { Secondary infrastructures }\end{array}$ & $\begin{array}{l}\text { - Information needs aggregation, } \\
\text { training, dissemination } \\
\text { Restricted or reserved control of } \\
\text { sensitive data production and use }\end{array}$ \\
\hline Components & $\begin{array}{l}\text { Descriptive documents and visual models } \\
\text { (analogical or digital) }\end{array}$ & $\begin{array}{l}\text { Integrated representation of policies and } \\
\text { visual model of the real-time status of city } \\
\text { data availability, planned operations, } \\
\text { uncovered areas (supported by digital } \\
\text { technologies) }\end{array}$ \\
\hline
\end{tabular}

\section{The City Data Plan}

The previous section analysed corporate data governance plans and urban plans to explain how their key components had been reinterpreted in defining the concept of the City Data Plan as a policy instrument for the governance of city data. Building on that, this section is going to synthetically present the CDP concept by reorganising and connecting the various aspects seen above in the two following clusters:

(a) Preparation and management of the CDP

(b) Contents of the CDP

The preparation and management of a City Data Plan require first of all the design of the institutions to get local stakeholders cooperating in the governance of city data, especially within smart city programmes. The CDP concept includes the four following institutions, described here under the responsibility assignment schema taken from corporate data governance plans.

The advisory board is led by the local authorities and composed of representatives of all local stakeholders interested to be recognised as part of the city data ecosystem. The advisors represent local organisations involved in smart city programmes in different roles, not only as data providers or data users, to participate and monitor the choices concerning city data governance arrangements. The composition of the advisory board varies over time, according to evolving needs and opportunities given by on-going projects and initiatives. The functions of the advisory board are establishing priorities and politically orienting the definition of targets and objectives to align the management of city data to the vision and development goals of the city. While the advisory board establishes the shared rules and principles for the city data governance and it is considered accountable for their implementation, this institution does not have operational functions. However, it is intended for the board to be consulted on major decisions requiring multi-stakeholder negotiations, but not on daily operations or intra-organisations data-related activities.

The teams of domain experts and data experts are constituted by urban area or sector of activity, and considered as distributed units affiliated with and representing the different stakeholders in that 
area or sector. They are responsible for surveying local information needs, available data sources, on-going data-related initiatives, and therefore for the implementation of the City Data Plan as a strategy to achieve specific formal goals for the development of the city through a cooperative governance of city data. At an operational level, each team is independent in establishing the functional goals to orient data operations among organisations that are interdependent in the processes of data generation, processing, use, or working in the same area. The members of each team, affiliated with the local organisations involved in the implementation of the CDP, independently work within their organisation aware of the common operational framework.

The coordination team can be established within a specific organisation (e.g., local government) or as a mixed group from different organisations (e.g., local government, university, foundations). The key function of the coordination team is supporting communication and enabling cooperative practices across the distributed units of domain and data experts. In particular, the coordination team has the responsibility to get the distributed teams consulted and informed in the cases the activities of other groups could impact on the established targets and agreements for a specific area, in compliance with the protocols decided by the Advisory board. On the other side, the coordination team is consulted and informed by the Advisory board in case of updates to the plan or preparation of sectorial subplan. The distributed team can consult and inform the coordination team about procedural conflicts to be addressed.

A unit of the coordination team, or a distinct dedicated group of data experts, is responsible for coordinating the emergency response across different organisations in the most severe crisis threatening city data resources, and addressing in a centralised way specific risks and issues related to data of public interest. The emergency team works in collaboration with the coordination team, by consulting specific distributed team when necessary, and informing the advisory board on the emergency response strategy.

All these institutions are intended as light-weight governance structures, with a marginal impact on the workload of the organisations involved in the implementation of the City Data Plan and not requiring any restructuring of their internal workflows. The aim of these institutions is maximising the benefits coming from the availability of city data among local organisations, and minimising data duplication efforts, extra-costs related to the mismanagement and low accessibility of data resources at the urban level, risks of malevolent uses of city data by local actors.

The contents of the City Data Plan consist of a set of dynamic map-based visualisations to support independent decisions of local organisations concerning their involvement in the process of generation and use of city data and the level of cooperation with other actors active in the same area or domain.

- The mapping of local organisations indicates their roles and levels of engagement in the city data ecosystem.

- The mapping of available data indicates the existing data sources covering each area of the city at various scales (e.g., metropolitan area, district, neighbourhood, building block) and on different domains (e.g., mobility, energy, community, urban development, business). These data sources include big, medium, and small data available under the conditions established by each organisation (e.g., free access, fees payment, data exchange) within the rules of the CDP. Updates on the available data sources can be independently shared on the map-based system by each organisation.

- The mapping of information needs and targets by area and domain consist of the list of data (small, medium, big) to be produced in the short, medium and long term, as part of the general CDP or sectorial sub-plans. The survey of the information needs of local organisations can be initiated in the preparatory phase of the City Data Plan (as part of the "primary infrastructuring activities") and then dynamically updated over time. The targets actively supported by the institutions contributing to the CDP are established by the advisory board in relation to the development goals of the city, and then detailed by the operational units. The description of information needs 
and targets is complemented by the list of incentives, disincentives, and data transfer mechanisms oriented to facilitate and support their fulfilment.

- The mapping of on-going data generation processes and applications of city data currently in use includes the list of actors participating in the process (by domain, area and scale) and the "rules of engagement" for third parties interested in entering in the process, reusing the produced resources, extending the data sources to other domains, areas, or scale of intervention.

- The mapping of archived data sources by owner, area and domain works as the index of inactive data resources, not in use, but potentially required for future uses. They can include, for instance, reports, projects, surveys, datasets not updated anymore.

As mentioned before, widespread tools such as Web-GIS integrated with crowdsourcing functionalities can provide the technological support to the construction, use and updates of the map-based information representing the City Data Plan. The description of the architecture of the specific information system of the City Data Plan is out of the scope of this work, but it is important to notice that this type of tools is nowadays frequently integrated into participatory urban planning practices.

Besides the multi-level mapping of data resources in the city, the contents of the CDP include policy documents containing:

- The list of principles and operational recommendations to separate IT governance from data governance in the agreements between data providers and data users, as well as between owners of IT resources and data services providers

- The criteria to benefit of the public or private incentives for the production of data meeting local information needs and city targets

- The criteria activating the enforcement of disincentive mechanisms and the list of limitations associated with the misuse of city data concerning the access to the CDP resources

- The conditions regulating data rights transfer of data of public interests, and the recommendations for equitable data exchange among different stakeholders in the city data ecosystem covering the roles of data subjects, data collectors, data processors, data users on one side, and the role of data providers on the other side

- The standardised communication protocols at an inter-organisational level among local organisations cooperating in the data generation and use in the same area or domain of activity

- The schema of competences and responsibilities of the institutions implementing the CDP, specifying the functioning of centralised decision-making processes at the city level, and the coordination mechanisms for decentralised operations at the level of single organisations

- The guidelines for conflicts resolution in data-related operations

- The code of conduct for the local organisations involved in the CDP institutions

\section{Examples of Urban Applications of a City Data Plan}

This section provides two examples of applicative scenarios in which the use of this type of policy instrument can orient data governance mechanisms among city stakeholders. The first example concerns data in the domain of urban mobility, one of the most interested by smart city initiatives. The second example covers instead the domains of social services, a theme across the axes of smart people, smart communities and smart living of smart city frameworks [62], but marginally represented in smart city programmes [63].

These scenarios are contextualised in the city of Milton Keynes (UK), where the author had been working in the last two years on understanding local needs and expectations associated with city data. In this context, findings of a previous work [64] indicated that there is a structural misalignment between data that are currently available or commonly produced in the city and data required by local stakeholders to pursue their goals in ordinary activities such as the delivery of public or private 
services, community initiatives, and business projects. Moreover, the different stakeholders involved in the city data ecosystem as organisations producing or interested to use city are not mapped or framed in shared institutions. Therefore, their relationships are not supported by clear rules or protocols that could facilitate reciprocal data exchange and collaborative practices related to data collection, analysis, use and dissemination. These issues can be observed in several other contexts and often impact on the successful implementation of smart city initiatives.

These findings emerge from a set of scenarios outlined in a workshop with local stakeholders held in Milton Keynes to specifically discuss of their information needs related to city data. The two scenarios reported below had been selected among the ones developed in the workshop to illustrate some basic applications of a City Data Plan in real settings.

Milton Keynes constitutes an interesting context to explore how the City Data Plan would contribute to establish a multi-stakeholder governance of city data compliant with the strategic vision of future smart initiatives and the development goals of the city. Indeed, Milton Keynes had been characterised in the past by a strong commitment of public authorities and privates in supporting innovation programmes and smart city initiatives [65].

The three scenarios reported in this section focus on defining the information needs of local stakeholders in relation to smart city visions and goals, aligning the production and use of data, and taking into account sensitive issues concerning privacy, equity, and accessibility of data.

\subsection{Scenario 1. Supporting Alternative Mobility Choices}

- Context. The combination of intense regional and internal mobility flows determine that traffic congestion is one of the most urgent problems that Milton Keynes is facing. Road congestion due to daily commuting has a strong negative impact on the environment and the perceived well-being of citizens.

- Smart City vision (Smart Mobility). Milton Keynes as city supporting slow and alternative mobility choices to actively reduce traffic levels and improve environmental sustainability at the urban level.

- Existing IT infrastructure and data sources: Traffic sensors monitoring about 300 roundabouts in the city and over 2000 parking sensors in the city centre.

- Physical constraints. Milton Keynes has two distinct road networks. The high-way system is reserved for cars and penetrates in residential compound through low-speed local streets. The bicycle and pedestrian network constituted by the so-called "red ways" is extended throughout the entire city, but scarcely used by residents and internal commuters. The population density is very low, and the urban area is over $90 \mathrm{sq} . \mathrm{km}$.

One of the ways to reduce traffic levels in Milton Keynes, at least at the neighbourhood scale, is by supporting cycle and pedestrian mobility on the red ways. However, the use of the red ways is particularly limited. To develop strategies and initiatives for incrementing their use among residents and internal commuters, the critical information that needs to be addressed concerns understanding the causes of the red ways underuse and what current uses could be incentivised and extended to more people.

Data coming from the existing traffic sensor network are not helpful to take decisions on these topics. On the other side, investments to extend the sensor infrastructure along the pedestrian paths are antieconomic because of the spatial extension of the red ways network, the low number of users concentrated in the weekends, privacy concerns raising specifically from the possibility to easily identify the red ways users (almost certainly residents in the same compound). More important, data coming from mobility sensors, while accurate and objective, do not address the information needs of local authorities interested in incentivising the use of red ways. Ad hoc surveys carried out on samples of the population are marginally helpful because of the variety of population profiles in each neighbourhood. 
In this scenario, a City Data Plan provides an overview of existing data sources (such as one of the traffic sensor networks), information needs of the mobility department of the local council and other organisations interested to sustainable mobility, and targets to reach in terms of coverage and depth of the required information. A CDP makes public and legible the established goal (sustainable cycle and pedestrian mobility) associated with specific information needs. Moreover, it enables local stakeholders to assess the consistency of potential measures put in place to fill the information gaps with the established goal. In this case, it makes understandable the implications of choosing to extend the sensor network to red ways (unsustainable investment, privacy risks, unfulfillment of the declared information need) to the advisory board and the relevant teams.

On the other side, the mapping of local organisations by area and domain of activity provides the opportunity to identify what organisations are the most likely to have relevant information to understand the underuse of red ways or their current uses. These organisations include, for instance, several sport and leisure associations regularly organising events and initiatives for their members. Even though these associations are not conventionally considered data collectors or data producers, actually they are and could be engaged to effectively achieve the CDP goals. Their "data resources", prevalently small data, can includes reports and formal documentation of past events, but also other untraced information that can be easily aggregated from a limited number of key persons rather than from a survey on population sample to have reliable insights on the problem to be addressed. The rules of engagement of civil society organisations by public or privates are contained in the CDP in terms of incentives, benefits, rights.

This example highlights how the institutional design instantiated in the CDP can serve goals related to smart mobility by working in synergy with local communities in the production of meaningful information in the public interest, without relying only on the IT infrastructure. The cooperative governance of city data results from working across smart city silos and enhancing the social capital, by maintaining transparency on goals and adopted protocols.

\subsection{Scenario 2. Monitoring the Impact of Social Services Provision on Local Communities}

- Context. Austerity measures and budgets cuts impose an attentive management of human resources in social and health service provision. On the other side, there is no decrease in the requests of assistance from areas characterised by high rates of socio-demographic vulnerability (due to poverty, unemployment, health issues).

- Smart City vision (Smart communities). Milton Keynes as a city where social and health services are planned and managed by monitoring and assessing the impact of area-based services.

- Existing data sources:

- $\quad$ Local Council. Data on the provision of social services over the years.

- $\quad$ National Health Service (NHS) facilities. Data on health services.

- $\quad$ Office for National statistics. Census data 2011, segmented by area.

- Public and private health professional associations (GP, pharmacists, nurses, homecare assistance). Data on the provided services.

- $\quad$ Local charities. Data on assisted people and beneficiaries of their activities.

- Organisational constraints. While communication protocols are established and partially formalised between local governments and regional and national public agencies as concerning data exchange, there are no standard measures in place to cooperate with non-profit and private organisations.

Despite the availably of data from multiple sources, the assessment of the impact of social and health services is challenging. Indeed, data produced by local council, national health system, and health professional associations are focused on the nature and quantity of the provided services. These data do not include inputs on the quality of life of the assisted customers and describe different segments of the population at different granularities, often in a fragmented way. Furthermore, Milton Keynes 
is a fast-growing city with a high rate of turnover of the population due to internal or international migration. Therefore, the demographic projection of census data collected every 10 years do not necessarily represent a reliable information base on the current socio-demographic characteristics by area.

As also seen in the previous scenario, on-field complementary information can be aggregated through experimental data collection initiatives in cooperation with local charities and other organisations. These initiatives can help the local council or public agencies in assessing the impact of their services. However, the central difference in this case is that a plurality of public and private have similar information needs and interests in monitoring and iteratively assessing the impact of their own resource management choices on the provided services.

In this scenario, the CDP is intended as a common framework to cooperatively support iterative data collection initiatives addressing shared information needs among multiple organisations. These information needs concern the structured analysis of factors hindering or contributing to the impact of social and health services at the local scale. Consensus on goals and targets is built within the advisory board. Then, functional goals and procedural aspects of this multi-organisation partnership are defined at the level of the coordination team. The management of the periodical operation of data collection and analysis is on mixed teams of domain experts (on social and health services, or on the specific context to be analysed) and data experts (to support the standardisation of the collected data).

The specific schema of responsibilities and competences can be elaborated within a sub-plan of the CDP, as well as the patterns of incentives, disincentives and data rights transfer to facilitate the data integration.

\section{Discussion}

The City Data Plan, as presented in this paper, is a theoretical construct described in its operational aspects, developed by using a design research approach. The intrinsic validity of the proposed concept relies on its compliance with the constraints posed in the problem framing (Section 3). The value of the CDP concept is formalising a potential solution to address part of the issues related to the governance of city data discussed in the literature review (Section 2).

The intrinsic validity of the proposed solution had been iteratively assessed during the development and refinement of the concept against the constraints made explicit at the beginning of the process. The key questions driving this assessment included:

- As regards Users: is the CDP an instrument able to address the needs and requirements of local stakeholders participating in the city data ecosystem in different roles? Does the CDP take into account their different organisational constraints (e.g., mission, core activities, human resources)? Can the CDP be integrated into existing processes?

- As regards city data as Object of the plan: is the CDP a policy instrument able to prevent or limit the manipulation of data by technology providers and their clients? Are the measures included in the CDP potentially effective in contrasting malevolent or discriminatory uses of data at the local level?

- As regards the Context of use of data by local stakeholders: is the CDP not in contrast with other laws and regulations? Can the CDP provide support to different types of decisions and actions in the city driven by data?

In the following section, we break down the arguments associated with these three sets of questions. 


\subsection{Users}

The concept of the City Data Plan extends the proposal developed by Kitchin to address the issues of data governance in smart cities [24] by elaborating what types of measures can frame and support the independent activities of different institutional structures in charge of orienting and managing data-related operations in the city.

In this regard, the organisational bodies associated with the preparation and implementation of the City Data Plan take into account the different missions and structures of the various classes of stakeholders in the city data ecosystem. The local government is expected to facilitate the activities of the advisory board and take the lead of the coordination team by virtue of its institutional mission and the associated legitimate competences. Public agencies and knowledge cluster are considered as subject operating in the city but independent from local government in pursuing their missions within the advisory board and through the distributed operational team. Non-profit organisations and civil society groups are included in every governance structure because of their privileged position in understanding local information needs and the phenomena in the context. Local business, not limited to technology companies, are considered as potential sources and users of information, even in the cases where data experts are not part of their human resources and data management is not their core activity. In any case, every type of stakeholder is assumed as potentially covering all the roles in the city data ecosystem, from data provider to data consumer, from data subject to data processor.

To limit the burden of participating in the preparation and implementation of the plan, the CDP envision distributed teams coordinated by a centralised unit. Each distributed unit (that can also correspond to just one referent per organisation) work independently from the others and within the structure and rules of the organisation it belongs. In this way, adhering to the CDP governance structures can be integrated into existing management processes. Indeed, a significant improvement of the current city data governance can be achieved by constructing a clear understanding of who produce what data, for what purpose, when, where, and under what conditions those data can be accessed, and by whom. Building this knowledge base and keeping it updated does not imply internal restructuring of local organisations. Then, the rules of engagement with the CDP, and the protocols for inter-organisational cooperation on data management are aimed to minimise uncertainties due to the variety of organisational structures in terms of communication protocols, data exchange measures, and use of shared resources.

\subsection{Object}

The CDP as a policy instrument is intended to prevent or limit the manipulation of data by making explicit the rationale and the political choices supporting the deployment of specific smart city technologies in urban environment and the collection of specific data sources. In other words, a CDP helps to depict the various elements constituting the "data assemblage" [27]: political, social, economic, environmental, organisational and technical factors impacting on the choices concerning data collection and analysis.

Indeed, the CDP is proposed as a common decision framework for every stakeholder in the city data ecosystem to assess the compatibility of data operations with the development goals of the city established in the advisory board. The negotiation process accompanying the CDP making is oriented to reach a consensus among city stakeholders on the contents of city data policies, and in particular on distinguishing between uses of data considered legitimate and illegitimate in a specific context.

The measures included in the CDP are expected to outline the rules of action and interactions with city data and other local stakeholders, contrasting malevolent or discriminatory uses of data at the local level through monetary and non-monetary disincentives described in previous sections.

The conceptualisation of the CDP focused on data produced by smart city technologies in a specific area and under specific agreements between technology providers and public authorities, as well as other data sources produced by local stakeholders that are fragmented, not easily reachable, not aggregated. An open issue not addressed by the City Data Plan concerns the production and use 
of city data managed by organisations not related to the city, such as major web-services providers like Google or Facebook. The risks associated with the manipulation and malevolent use of data by these actors need to be addressed at upper level rather than at municipal level.

\subsection{Context}

The City Data Plan is envisioned as a policy instrument that can integrate or complement existing regulations, laws and policies concerning both data and urban resources. In particular, urban laws and regulations defined at a city level can be extended to include specific section and measures concerning city data considered as urban resources of public interest, even when they are owned by privates and managed according to market-driven mechanisms. Concerning aspects already addressed by existing norms, such as privacy issues and the GDPR [7], a City Data Plan can provide an orientation grid to navigate the different types of concerns associated with different types of data at various spatial and temporal granularities. This form of support can facilitate a pre-assessment of the normative compliance of smart city initiatives and the related production and use of data.

A City Data Plan can also be seen as a "smart law" in itself, following the definition proposed by Decker [39]. Indeed, the particular form, structure and management of an urban plan give to this type of instrument the flexibility to distinguish between high-level principles and decisions on one side, and on the other side low-level operational aspects related to the management of data. The operational protocols can be dynamically revised according to the evolution of technologies and local needs and their effects on city activities, nature and characteristics of data, and definition of data-centred services. Nevertheless, contingent and dynamic variations remain bordered within the coherent decision framework constituted by the CDP.

The high-level and low-level principles, schemas, and implementation mechanisms of the CDP are structured around the types of decisions and actions of local stakeholder. These decisions include strategic and long-term decisions, resource decisions at medium term, and resources allocation decision at short terms. To adequately support these different types of decisions, the CDP enlarges the spectrum of data considered in the plan to small and medium data, and not only big data commonly associated with smart city technologies. In addition, the CDP establishes shared rules also for private, internal, public, restricted data, and not only open data commonly considered as the only data resources of public interest. Indeed, for instance, the insights to guide strategic decisions at the local level can also come from informal sources or previous analysis contained in documents classified as small data. Moreover, the licensing of data needs to be considered as a property independent from the value or the relevance of the required information for a specific type of decision. The CDP enables each stakeholder to decide if the value of a certain dataset justifies their acquisition by payment of a fee, or if other available options (mapped in the plan) can constitute valid alternative sources of information for the type of decision they have to make.

\subsection{Open Challenges}

This discussion highlighted how the CDP concept meets the five meta-requirements established as the starting point of this work and the arguments supporting its intrinsic validity. Two more points necessitate further clarifications.

Political openness and commitment to establish a cooperative governance of data at the city level are the preconditions for preparing a City Data Plan, as well as the interest and active engagement of city stakeholders to participate in the process. However, these two points constitute the preconditions for defining and implementing every other type of urban policy, independently from the addressed topic. In the case of the governance of city data and the alignment of smart city initiatives to the vision and goals of the city, important drivers are the growing awareness of the public opinion on the consequences of an unregulated use of data and the willingness of local governments to regain independence and autonomy from technology providers, as mentioned in Section 1 . Therefore, the ideal 
testbed to implement a City Data Plan is an urban context characterised by a strong political leadership and cohesive active social forces reclaiming people-centred smart city vision for the future of their city.

From a practical perspective, the preparation of a CDP requires a team of professionals with different expertise to guide the activities and concretely realise the plan. The team should include planners, policy designers, data scientists, information system analysts and legal experts. Differently from the development of an urban plan-which can be managed by the internal staff of the planning department of local authorities or by external planning agencies-the preparation of a CDP implies temporarily recruiting specialists for this purpose, facilitating reciprocal multi-disciplinary understanding, and coordinating the team agenda with the activities in collaboration with city stakeholders (such as setting up the advisory board, the distributed teams in local organisations, the emergency units). However, this type of challenge is common within service design processes or social innovation design initiatives, both in the public and private sector. Experiences and inputs from these fields can support the prototyping of standardised, effective and easily manageable CDP making processes.

Future works will extend and further detail the conceptualisation of the City Data Plan by focusing on the functioning of the organisational bodies associated with the CDP and investigating the procedural aspects of realising the CDP in real-world settings.

\section{Conclusions}

The City Data Plan concept described in this paper can be summarised in the following points. A CDP:

- Is a policy instrument that can have a regulatory, statutory or consultative value, in compliance with the conventions of a specific normative context

- Has the form of an inter-organisational set of protocols defining goals, responsibilities, and operations on city data

- Provides a common decision framework for public and privates organisations to support city data production and use, based on local information needs/resources

- Establishes communication procedures to coordinate the city stakeholders involved in the city data ecosystem in different roles

- Includes a set of mechanisms regulating the plan implementation through incentives, disincentives, and rights transfer agreements

- Can be instantiated in a synthetic visual model mapping information needs, available or required data resources, on-going data collection initiatives, and data-in-use applications

The goal of the proposed concept is to outline a potential path for aligning the production and use of data generated by smart city technologies with the visions and goals set for the development of the city. The idea of making a plan to support a cooperative governance of city data indeed provides a possible answer to the two challenges stated in the introduction, Section 1. A City Data Plan is an instrument able to frame the production and use of city data into a comprehensive vision of social and economic local development driven by the equitable access to these resources to all local stakeholders. Secondarily, the measures and implementation mechanisms of a CDP can facilitate a socially and economically sustainable redistribution of the benefits associated with the availability of an unprecedented amount of data on every aspect of city life.

A plan of city data can help to manage social conflicts due to the inequalities and unbalanced power relationships between those who exploit data and those who are exploited for data and through data. Indeed, its preparation and the rules established in the CDP provide legibility and support to interactions in the city data ecosystem. Furthermore, differently from other data governance solutions focused on individuals, the development and implementation of a City Data Plan are grounded on the fact that organisations are the essential entities to innovate the city data governance. Local organisations are indeed deeply affected or empowered by the production and use of data more than individuals. 
The contribution of the CDP concept to the problem of the city data governance in smart cities is envisioning a pragmatic and flexible approach to setting up light-weight negotiation protocols to improve trust and cooperation among the various local stakeholders involved directly and indirectly in the city data ecosystem. These negotiation protocols are included in familiar and widely accepted policy instruments such as general urban plans that traditionally recognise the legitimate interests of local stakeholders in exploiting urban resources contributing to the development of the city.

The contribution of this work to the urban planning research and practice is two-fold. First, it extends the analysis of the themes covered by urban plans to the new "data layer" generated by smart city technologies and data-related actions in the city [13,14], outlining a research space for further empirical and theoretical investigations on this topic in planning research. Second, the prototyping and implementation of City Data Plans can be interpreted as future professional paths for an active transfer of urban planning practices to the applicative domain of smart city programmes. In this sense, the development of the CDP concept is an example of knowledge transfer between these two domains-urban planning and smart city-in which urban planning knowledge is recognised as generative for new solutions to current problems posed by the deployment of smart city technologies in urban environments.

Funding: This research received no external funding.

Acknowledgments: I would like to thank Alessio Antonini for the long conversations about the concept presented in this paper, but also the Open University and the KMi for the access to the library resources during my visiting period in these institutions.

Conflicts of Interest: The author declares no conflict of interest.

\section{References}

1. Abella, A.; Ortiz-De-Urbina-Criado, M.; De-Pablos-Heredero, C. A model for the analysis of data-driven innovation and value generation in smart cities' ecosystems. Cities 2017, 64, 47-53. [CrossRef]

2. Barns, S. Smart cities and urban data platforms: Designing interfaces for smart governance. City Culture Soc. 2018, 12, 5-12. [CrossRef]

3. Kitchin, R. The Data Revolution: Big Data, Open Data, Data Infrastructures and Their Consequences; Sage: Thousand Oaks, CA, USA, 2014.

4. Martínez-Ballesté, A.; Pérez-Martínez, P.A.; Solanas, A. The pursuit of citizens' privacy: A privacy-aware smart city is possible. IEEE Commun. Mag. 2013, 51, 136-141. [CrossRef]

5. Sadowski, J.; Pasquale, F.A. The spectrum of control: A social theory of the smart city. First Monday 2015, 20. Available online: https://ssrn.com/abstract=2653860 (accessed on 30 May 2019). [CrossRef]

6. Van Zoonen, L. Privacy concerns in smart cities. Gov. Inf. Q. 2016, 33, 472-480. [CrossRef]

7. GDPR. Available online: https://eugdpr.org/ (accessed on 20 July 2019).

8. Data as Commons. Available online: https://ajuntament.barcelona.cat/digital/en/digital-transformation/citydata-commons (accessed on 30 May 2019).

9. TADA Manifesto. Available online: https://tada.city/en/home-en/ (accessed on 20 July 2019).

10. Thorns, D.C. The Transformation of Cities: Urban Theory and Urban Life; Macmillan International Higher Education: London, UK, 2017.

11. Mazza, L. Technical Knowledge, Practical Reason and the Planner's Responsibility. Town Plan. Rev. 1995, 66, 389-410. [CrossRef]

12. Hoch, C. Planning craft: How planners compose plans. Plan. Theory 2009, 8, 219-241. [CrossRef]

13. Anthopoulos, L.G.; Vakali, A. Urban Planning and Smart Cities: Interrelations and Reciprocities. In The Future Internet; Springer: Berlin/Heidelberg, Germany, 2012; pp. 178-189.

14. Townsend, A. Cities of data: Examining the new urban science. Public Culture 2015, 27, 201-212. [CrossRef]

15. Fernandez-Anez, V.; Fernández-Güell, J.M.; Giffinger, R. Smart City implementation and discourses: An integrated conceptual model. The case of Vienna. Cities 2018, 78, 4-16. [CrossRef]

16. Yigitcanlar, T.; Kamruzzaman, M.; Buys, L.; Ioppolo, G.; Sabatini-Marques, J.; da Costa, E.M.; Yun, J.J. Understanding 'smart cities': Intertwining development drivers with desired outcomes in a multidimensional framework. Cities 2018, 81, 145-160. [CrossRef] 
17. Allam, Z.; Newman, P. Redefining the smart city: Culture, metabolism and governance. Smart Cities 2018, 1, 4-25. [CrossRef]

18. Gunder, M.; Madanipour, A.; Watson, V. (Eds.) The Routledge Handbook of Planning Theory; Routledge: Abingdon-on-Thames, UK, 2017.

19. Healey, P. Urban Complexity And Spatial Strategies: Towards A Relational Planning For Our Times; Routledge: Abingdon-on-Thames, UK, 2006.

20. Cowley, R.; Joss, S.; Dayot, Y. The smart city and its publics: Insights from across six UK cities. Urban Res. Pract. 2018, 11, 53-77. [CrossRef]

21. Yigitcanlar, T. Planning for knowledge-based urban development: Global perspectives. J. Knowl. Manag. 2009, 13, 228-242. [CrossRef]

22. Taylor, L.; Richter, C. Big data and urban governance. In Geographies of Urban Governance; Springer: Cham, Switzerland, 2015; pp. 175-191.

23. Barns, S. Mine your data: Open data, digital strategies and entrepreneurial governance by code. Urban Geogr. 2016, 37, 554-571. [CrossRef]

24. Kitchin, R. Getting Smarter about Smart Cities: Improving Data Privacy And Data Security; Data Protection Unit, Department of the Taoiseach: Dublin, Ireland, 2016.

25. Pan, Y.; Tian, Y.; Liu, X.; Gu, D.; Hua, G. Urban big data and the development of city intelligence. Engineering 2016, 2, 171-178. [CrossRef]

26. Barns, S.; Cosgrave, E.; Acuto, M.; Mcneill, D. Digital infrastructures and urban governance. Urban Policy Res. 2017, 35, 20-31. [CrossRef]

27. Kitchin, R. Data-Driven, Networked Urbanism. 2015. Available online: https://ssrn.com/abstract=2641802 (accessed on 30 May 2019). [CrossRef]

28. Kitchin, R. The real-time city? Big data and smart urbanism. GeoJournal 2014, 79, 1-14. [CrossRef]

29. Shelton, T.; Zook, M.; Wiig, A. The 'actually existing smart city'. Camb. J. Reg. Econ. Soc. 2015, 8, 13-25. [CrossRef]

30. McNeill, D. Global firms and smart technologies: IBM and the reduction of cities. Trans. Inst. Br. Geogr. 2015, 40, 562-574. [CrossRef]

31. Kietzmann, J.H.; Hermkens, K.; McCarthy, I.P.; Silvestre, B.S. Social media? Get serious! Understanding the functional building blocks of social media. Bus. Horiz. 2011, 54, 241-251. [CrossRef]

32. Lee, C.S.; Long, M. News sharing in social media: The effect of gratifications and prior experience. Comput. Hum. Behav. 2012, 28, 331-339. [CrossRef]

33. Hampton, K.N.; Rainie, H.; Lu, W.; Dwyer, M.; Shin, I.; Purcell, K. Social Media and the 'Spiral of Silence'; PewResearchCenter: Washington, DC, USA, 2014.

34. Harris, R.; Sleight, P.; Webber, R. Geodemographics, GIS and Neighbourhood Targeting; John Wiley \& Sons: Hoboken, NY, USA, 2005; Volume 8.

35. Bowker, G.C. Big data, big questions| the theory/data thing. Int. J. Commun. 2014, 8, 5.

36. Leszczynski, A. Speculative futures: Cities, data, and governance beyond smart urbanism. Environ. Plan. A Econ. Space 2016, 48, 1691-1708. [CrossRef]

37. Lyon, D. Surveillance, Snowden, and big data: Capacities, consequences, critique. Big Data Soc. 2014, 1, 1-11. [CrossRef]

38. Finch, K.; Tene, O. Welcome to the metropticon: Protecting privacy in a hyperconnected town. Fordham Urb. Law J. 2013, 41, 1581.

39. Decker, A. Smart Law for Smart Cities. Fordham Urb. Law J. 2016, 41, 1491.

40. Rabari, C.; Storper, M. The digital skin of cities: Urban theory and research in the age of the sensored and metered city, ubiquitous computing and big data. Camb. J. Reg. Econ. Soc. 2014, 8, 27-42. [CrossRef]

41. Ting, L.; Williamson, I.P. Spatial data infrastructures and good governance: Frameworks for land administration reform to support sustainable development. In Proceedings of the 4th Global Spatial Data Infrastructure Conference, Cape Town, South Africa, 13-15 March 2000; pp. 13-15.

42. Cross, N. From a design science to a design discipline: Understanding designerly ways of knowing and thinking.". In Design Research Now; Birkhäuser: Basel, Switzerland, 2007; pp. 41-54.

43. Kolko, J. Abductive thinking and sensemaking: The drivers of design synthesis. Des. Issues 2010, 26, 15-28. [CrossRef]

44. Thomas, G. The DGI Data Governance Framework; The Data Governance Institute: Orlando, FL, USA, 2006. 
45. Wende, K. A model for data governance-Organising accountabilities for data quality management. In Proceedings of the 18th Australasian Conference on Information Systems, Toowoomba, Australia, 5-7 December 2007.

46. Otto, B. A morphology of the organisation of data governance. In Proceedings of the European Conference on Information Systems (ECIS), Helsinki, Finland, 9-11 June 2011; Volume 20, p. 1.

47. Pierce, E.; Dismute, W.S.; Lwanga Yonke, C. The State of Information and Data Governance-Understanding How Organizations Govern Their Information and Data Assets; International Association for Information and Data Quality (IAIDQ); University of Arkansas at Little Rock, Information Quality Program (UALR-IQ): Baltimore, MD, USA; Little Rock, AR, USA, 2008.

48. Cheong, L.K.; Chang, V. The need for data governance: A case study. In Proceedings of the 18th Australasian Conference on Information Systems, Toowoomba, Australia, 5-7 December 2007.

49. Khatri, V.; Brown, C.V. Designing data governance. Commun. ACM 2010, 53, 148-152. [CrossRef]

50. Weber, K.; Otto, B.; Österle, H. One size does not fit all-A contingency approach to data governance. J. Data Inf. Qual. (JDIQ) 2009, 1, 4. [CrossRef]

51. Wakefield, J. The Google City That Has Angered Toronto. Available online: https://www.bbc.co.uk/news/ technology-47815344 (accessed on 25 July 2019).

52. Wende, K.; Otto, B. A Contingency Approach to Data Governance. In Proceedings of the 12th International Conference on Information Quality, Cambridge, MA, USA, 9-11 November 2007; pp. 163-176. Available online: https://www.alexandria.unisg.ch/213308/ (accessed on 30 May 2019).

53. Frischmann, B.M.; Madison, M.J.; Strandburg, K.J. (Eds.) Governing Knowledge Commons; Oxford University Press: Oxford, UK, 2014.

54. Open Data Institute. The Data Spectrum Helps you Understand the Language of Data. Available online: https://heodi.org/about-the-odi/the-data-spectrum/ (accessed on 30 May 2019).

55. Janin Rivolin, U. Planning systems as institutional technologies: A proposed conceptualization and the implications for comparison. Plan. Pract. Res. 2012, 27, 63-85. [CrossRef]

56. Punter, J.; Carmona, M. The Design Dimension of Planning: Theory, Content, and Best Practice for Design Policies; Taylor \& Francis: Abingdon-on-Thames, UK, 1997.

57. Available online: https://www.gov.uk/government/collections/planning-practice-guidance (accessed on 30 May 2019).

58. Healey, P. Collaborative Planning: Shaping Places in Fragmented Societies; Macmillan International Higher Education: London, UK, 1997.

59. Sandercock, L. Negotiating fear and desire. In Urban Forum; Springer: Amsterdam, The Netherlands, 2000; Volume 11, pp. 201-210.

60. Hopkins, L.D. Urban Development: The Logic of Making Plans; Island Press: Washington, DC, USA, 2001; Volume 166.

61. Micelli, E. La Gestione Dei Piani Urbanistici: Perequazione, Accordi, Incentivi; Marsilio Editori Spa: Venezia, Italy, 2012.

62. Chourabi, H.; Nam, T.; Walker, S.; Gil-Garcia, J.R.; Mellouli, S.; Nahon, K.; Pardo, T.A.; Scholl, H.J. Understanding smart cities: An integrative framework. In Proceedings of the 2012 45th Hawaii International Conference On System Sciences, Maui, HI, USA, 4-7 January 2012; pp. 2289-2297.

63. Alizadeh, T. An investigation of IBM's Smarter Cites Challenge: What do participating cities want? Cities 2017, 63, 70-80. [CrossRef]

64. Lupi, L.; Alessio, A.; De Liddo, A.; Motta, E. Actionable Open Data: Connecting City Data to Local Actions. Available online: https://arxiv.org/submit/2715230/view (accessed on 30 June 2019). [CrossRef]

65. Caprotti, F.; Cowley, R.; Flynn, A.; Joss, S.; Yu, L. Smart-Eco Cities in the UK: Trends and City Profiles 2016; University of Exeter: Exeter, UK, 2016.

(C) 2019 by the author. Licensee MDPI, Basel, Switzerland. This article is an open access article distributed under the terms and conditions of the Creative Commons Attribution (CC BY) license (http://creativecommons.org/licenses/by/4.0/). 